\title{
Chemistry induced by the impacts: Observations
}

\author{
By EMMANUEL LELLOUCH
}

\author{
Observatoire de Paris, 92195 Meudon, France
}

\begin{abstract}
This paper reviews spectroscopic measurements relevant to the chemical modifications of Jupiter's atmosphere induced by the Shoemaker-Levy 9 impacts. Such observations have been successful at all wavelength ranges from the UV to the centimeter. At the date this paper is written, newly detected or enhanced molecular species resulting from the impacts include $\mathrm{H}_{2} \mathrm{O}, \mathrm{CO}, \mathrm{S}_{2}, \mathrm{CS}_{2}, \mathrm{CS}, \mathrm{OCS}, \mathrm{NH}_{3}, \mathrm{HCN}$ and $\mathrm{C}_{2} \mathrm{H}_{4}$. There is also a tentative detection of enhanced $\mathrm{PH}_{3}$ and a controversial detection of $\mathrm{H}_{2} \mathrm{~S}$. All new and enhanced species were detected in Jupiter's stratosphere. With the exception of $\mathrm{NH}_{3}$ (and perhaps $\mathrm{H}_{2} \mathrm{~S}$ and $\mathrm{PH}_{3}$ ), apparently present down to the 10-50 mbar level, the minor species are seen at pressures lower than 1 mbar or less, consistent with a formation during the plume splashback at 1-100 microbar. $\mathrm{NH}_{3}$ may result from upwelling associated with vertical mixing generated by the impacts. The main oxygen species is apparently $\mathrm{CO}$, with a total mass of a few $10^{14} \mathrm{~g}$ for the largest impacts, consistent with that available in $400-700 \mathrm{~m}$ radius fragments. The observed $\mathrm{O} / \mathrm{S}$ ratio is reasonably consistent with cometary abundances, but the $\mathrm{O} / \mathrm{N}$ ratio (inferred from $\mathrm{CO} / \mathrm{HCN}$ ) is much larger, suggesting that another $\mathrm{N}$ species was formed but remained undetected, presumably $\mathrm{N}_{2}$. The time evolution of $\mathrm{NH}_{3}, \mathrm{~S}_{2}, \mathrm{CS}_{2}$ shows evidence for photochemical activity taking place during and after the impact week.
\end{abstract}

\section{Introduction and brief overview of measurements}

The comet Shoemaker Levy-9 impacts on Jupiter on July 16-22, 1994 have produced a variety of impressive phenomena observable at different wavelengths that provided insight into the various phases of the event (meteor, fireball, plume evolution and re-entry) and the subsequent dynamical evolution of Jupiter's atmosphere. These observations and their interpretation are reviewed in several chapters of this volume. The present chapter is concerned with a particular aspect of the phenomenon, namely the observed chemical modifications of Jupiter's atmosphere induced by the impacts. The ultimate goal is to provide a list of the molecular species that were detected (either detected for the first time in Jupiter, or observed to be enhanced) subsequent to the impacts, with preliminary, but as reliable as possible, baseline estimates of their mixing ratios and total abundances.

The chapter is organized as follows. In the rest of this introductory section, a brief overview of the different types of measurements is given, including some remarks on their respective capabilities and the possible complications associated with their analysis. Then, the bulk of the paper (Sections 2 to 5 ) is dedicated to spectroscopic measurements performed during the impact week and within the next few weeks. For each minor species, the relevant measurements are described and compared, and differences are discussed. In some instances, a few suggestions are proposed which may help reconcile the different measurements. The long-term evolution is addressed in Section 6 . In Section 7, a synthesis of the observations of minor species is attempted, and some elementary conclusions are drawn. The entire chapter is focussed on molecular gaseous species. In particular, observations of atomic species at visible and UV wavelengths are not addressed, neither is the compositional characterization of aerosols. These two topics are discussed in the chapters by Crovisier and West, respectively. In addition, although some description on the modification of Jupiter's thermal structure (as inferred from $\mathrm{CH}_{4}$ 
and $\mathrm{C}_{2} \mathrm{H}_{2}$ infrared measurements) is occasionally needed when discussing abundance retrievals, this is not done here in a systematic way; we refer the reader to the chapter by Conrath for a complete description of the subject. Similarly, $\mathrm{H}_{2}$ and $\mathrm{H}_{3}^{+}$observations, which are relevant to magnetospheric rather than chemical phenomena, are not covered at all here (see chapter by Ip).

Measurements of minor species after the impacts have been successful at all wavelengths. In the UV, the most useful measurements come from the HST/FOS and GHRS spectra, which cover altogether the $1250-3300 \AA$ range. A preliminary analysis of these data was given by Noll et al. (1995) and more detailed modelling of the 1800-2300 range was performed by Atreya et al. (1995) and Yelle and McGrath (1996). The HST spectra show absorption features, from which $\mathrm{S}_{2}, \mathrm{CS}_{2}$ and $\mathrm{NH}_{3}$ have been unambiguously identified, and emission features, due to CS and to a number of atomic lines. IUE observations (Harris et al. 1995) also indicate the presence of ammonia absorption lines, but these measurements have not been analyzed yet. Modelling the absorption lines is primarily complicated by (i) the influence of scattering by the aerosols produced by the impacts; in particular the aerosol unit optical depth level in the UV is uncertain by about one order of magnitude in pressure (ii) line saturation effects: laboratory data do not resolve individual lines in the electronic bands, and curve-of-growth effects may be difficult to assess; in some cases $\left(e . g ., \mathrm{S}_{2}\right.$ ), available laboratory data are still preliminary. On the other hand, the determination of molecular abundances from UV absorption lines (i.e., in the solar reflected component) is not directly sensitive to the temperature, although the absorption cross sections may be significantly temperature-dependent (e.g., $\mathrm{S}_{2}$ ). For the emission lines, the chief problem at the present time is to ascertain the excitation mechanism. Noll et al. (1995) have assumed that they are produced by resonant fluorescence, but other mechanisms (notably non-resonant fluorescence (Carpenter et al. 1995) and thermal emission) may be at work and need to be further explored.

Infrared spectroscopic observations can be conveniently classified into two types of measurements: (i) the "plume/splashback phase" observations, i.e., the measurements obtained within $\sim 1 / 2$ hour from impact time, and (ii) the "impact site" observations, that are performed hours or days after the impacts, when the site material is dragged by Jovian rotation. All detections of minor species at infrared wavelengths were obtained in thermal, as opposed to solar reflected, radiation. Plume/splashback observations were achieved notably in the 2-3 $\mu \mathrm{m}$ range by Galileo/NIMS and many Earth-based telescopes (e.g., Anglo-Australian Telescope (AAT), Steward Observatory, Calar Alto) and at 720 microns from the KAO. These measurements are particularly difficult to analyze in terms of minor constituent abundance because: (i) they are extremely sensitive to temperature, as they correspond to the tail of the Planck function (especially at 2-3 $\mu \mathrm{m}$ ), (ii) their very special geometry (a relatively warm plume at the limb expanding in a colder environment) requires elaboration of unusual and complex models, and (iii) they are affected by complications associated with departure from local thermodynamical conditions (LTE); for example, in the case of the widely observed CO $2.3 \mu \mathrm{m}$ bands, non-LTE effects become important at pressures as deep as $\sim 1 \mu$ bar (Hooker and Millikan 1963). Site observations do not pose these problems so acutely and their analysis is similar to that of "classical" Jupiter observations, although knowledge of the temperature structure is an important aspect that must be coped with. Infrared site observations were successfully obtained at $4.7 \mu \mathrm{m}$ (CFHT/FTS, UKIRT/CGS-4, IRTF/CSHELL) and in the $8-12 \mu \mathrm{m}$ window. The latter observations have the advantage that the temperature profile can be measured "simultaneously" from the emission of hydrocarbons such as $\mathrm{CH}_{4}$ and $\mathrm{C}_{2} \mathrm{H}_{2}$, whose abundance is predicted to remain roughly unchanged during the fireball/splashback shock chemistry and subsequent photochemistry, and which therefore 


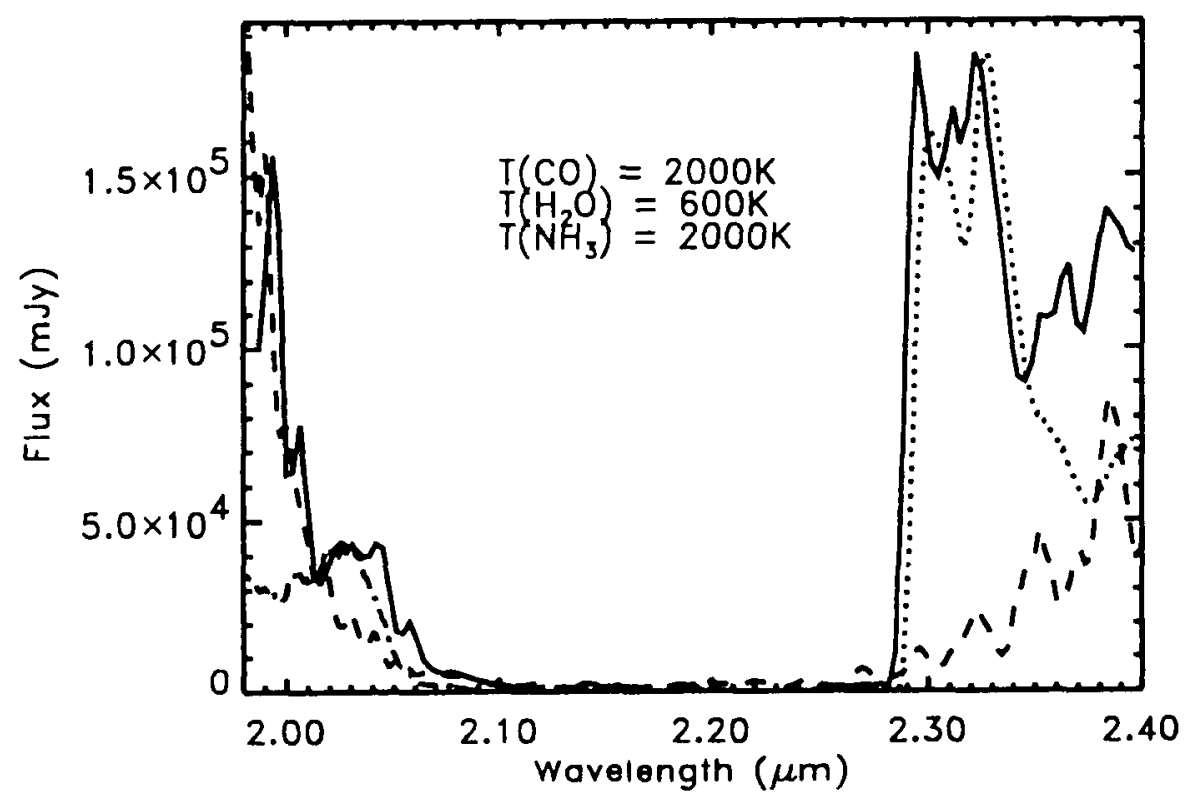

FIGURE 1. AAT/IRIS spectrum of fragment $\mathrm{K}$ splashback (continuum subtracted) along with a model including $\mathrm{H}_{2} \mathrm{O}$, $\mathrm{CO}$, and $\mathrm{NH}_{3}$. From Meadows and Crisp (1995a).

can be used as thermal probes of the stratosphere. Many successful results have been obtained at 8-12 $\mu \mathrm{m}$ from various types of experiments. Among these, one can mention (i) the heterodyne observations (IRTF/IRHS, Mount Wilson), which resolve the line profiles, thereby constraining the vertical profile of the minor compounds, although some complications arise because rotational broadening due to velocity smearing within the sites contributes to the lineshape and (ii) the spectro-imagers (in particular IRSHELL at the IRTF), which combine high spectral $(\sim 10000)$ and high spatial $\left(1-1.5^{\prime \prime}\right)$ resolution and provide unique information on the horizontal distribution of the minor species.

Finally, millimeter and centimeter heterodyne observations, performed at IRAM $(30-\mathrm{m})$, JCMT (15-m), SEST (15-m) and Medicina (32-m) have also allowed the detection of several new molecular species in Jupiter's stratosphere. In spite of the well-known weak (linear) dependence of the flux with temperature at these wavelengths, the analysis of these data is not insensitive to the thermal profile, because the lines (emission or absorption) are observed against a continuum whose brightness temperature is unfortunately very close (e.g., $170 \mathrm{~K}$ at $1.3 \mathrm{~mm})$ to the temperature of Jupiter's stratosphere. These observations are also hampered by the lack of spatial resolution ( $10^{\prime \prime}$ at best, more typically $20-30^{\prime \prime}$ ). As for the $10 \mu \mathrm{m}$ measurements, the lineshapes are resolved, but again rotational effects within the sites may be important; in addition, the spectra often mix the contribution from different sites.

\section{Oxygen species}

\subsection{Water}

One of the last molecules whose detection was announced, water has in fact been reported independently by five different teams. Four of these detections were obtained in the plume/splashback phase. Galileo/NIMS observed emission from the $2.7 \mu \mathrm{m}$ water band on impacts $\mathrm{G}$ and $\mathrm{R}$ (Carlson et al. 1995a). Observations with AAT/IRIS de- 


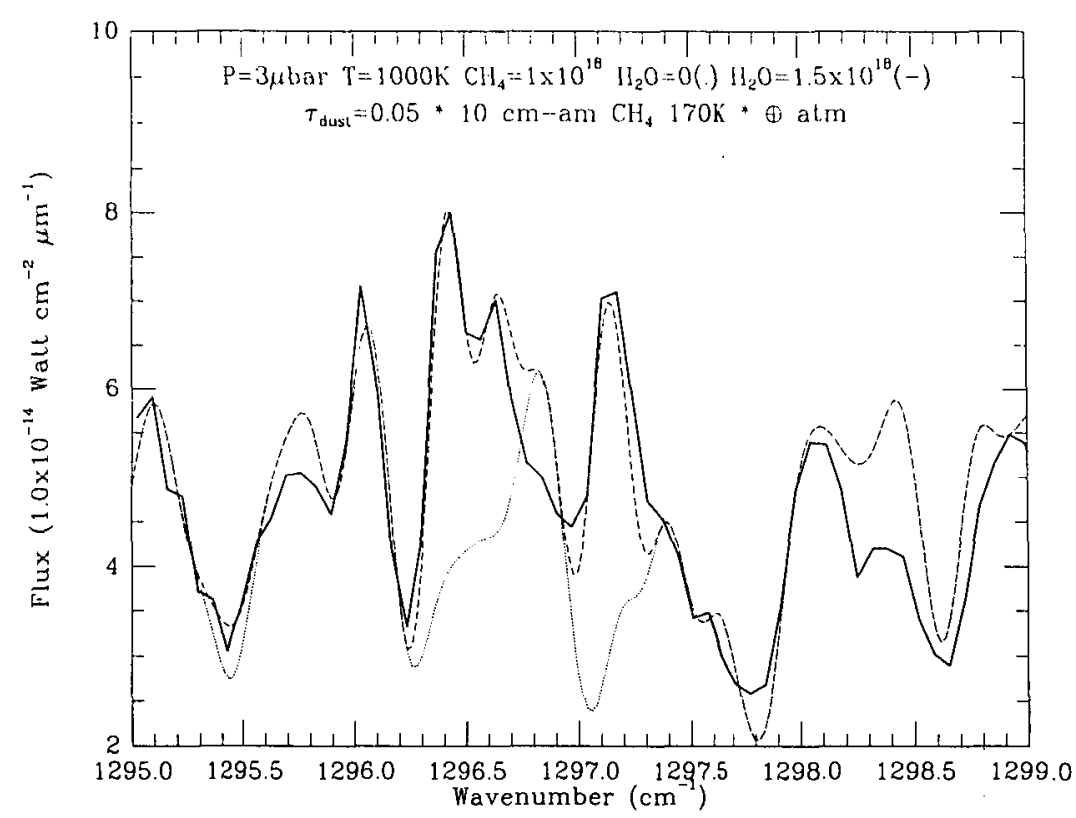

FIGURE 2. KAO/KEGS spectrum of impact $\mathrm{G}$ splashback, 14 minutes after impact (solid line). The $\mathrm{H}_{2} \mathrm{O}$ lines at 1296.4, 1296.7, and $1297.2 \mathrm{~cm}^{-1}$ are visible. Dotted line: model fit (see text) with $\mathrm{H}_{2} \mathrm{O}=0$. Dashed line: model fit, with a $\mathrm{H}_{2} \mathrm{O}$ column density $=1.5 \times 10^{18} \mathrm{~cm}^{-2}$. From Bjoraker et al. (1995a).

tected $\mathrm{H}_{2} \mathrm{O}$ at $1.99-2.02$ and $2.35-2.40$ micron on several $(\mathrm{C}, \mathrm{D}, \mathrm{G}, \mathrm{K}, \mathrm{R}, \mathrm{W})$ impacts (Meadows and Crisp 1995a; Fig. 1). In all cases, the observations correspond to 10 to 20 minutes after impact time $\left(\mathrm{T}_{i}\right.$, as defined, for example, by the moment of first signal on the Galileo instruments), and the water signal appeared to be delayed with respect to hot methane emission at 2.2 and $3.3 \mu \mathrm{m}$. Analysis of the NIMS data on impact $\mathrm{G}$, using a model described below (Bjoraker et al. 1995a), indicated a water mixing ratio of $100 \mathrm{ppm}$ at $\mathrm{p} \leq 3 \mu$ bar and $\mathrm{T}=2000 \mathrm{~K}$, corresponding to a total mass of $10^{12} \mathrm{~g}$; a factor-of-ten lower mass was inferred for impact $\mathrm{R}$ (Carlson et al. 1995b). Finally, the NIMS data on impact $\mathrm{G}$ also suggested a tentative detection of $\mathrm{H}_{2} \mathrm{O}$ at $2.7 \mu \mathrm{m}$ in the late fireball phase, $\sim 30-40 \mathrm{sec}$ after impact (Carlson et al. 1995c).

At longer wavelengths, $\mathrm{H}_{2} \mathrm{O}$ was unambiguously detected by the two experiments flown onboard the KAO. The high resolution $(\mathrm{R}=9000)$ instrument KEGS detected several $\mathrm{H}_{2} \mathrm{O}$ lines at 22.6 and $23.9 \mu \mathrm{m}$ and three others near $7.7 \mu \mathrm{m}(1296.4,1296.7$, and $1297.2 \mathrm{~cm}^{-1}$ ) on impacts $\mathrm{K}$ and $\mathrm{G}$ (Bjoraker et al. 1995a, 1995b; Fig. 2.). All of these lines have high lower energy levels $\left(500-700 \mathrm{~cm}^{-1}\right.$ at $22-24 \mu \mathrm{m}$ and $1500-2000 \mathrm{~cm}^{-1}$ at $8 \mu \mathrm{m})$. The lower resolution $(\mathrm{R}=300)$ instrument HIFOGS observed part of the water $\nu_{2}$ band near $6.6 \mu \mathrm{m}$ on impacts $\mathrm{R}$ and $\mathrm{W}$ (Sprague et al. 1996). The $22-24 \mu \mathrm{m}$ lines were visible in emission for about an hour after impact $\mathrm{G}$. At $6.6 \mu \mathrm{m}$ and $7.7 \mu \mathrm{m}$, the emissions appeared $\sim 5$ minutes after impact, peaked at $\sim \mathrm{T}_{i}+12-14$ minutes, and disappeared after another five minutes or so (Fig. 3). The duration of the emission correlates with the excitation level of the transition (Bjoraker et al. 1995b), strongly suggesting that the disappearance is primarily caused by the cooling of the stratosphere following the splashback-induced heating to several thousands of degrees. The $7.7 \mu \mathrm{m}$ range also showed hot lines of $\mathrm{CH}_{4}$. Modelling of the 6.6 and 7.7 micron spectra was performed in limb geometry, by assuming that the methane and water emissions are produced in a 


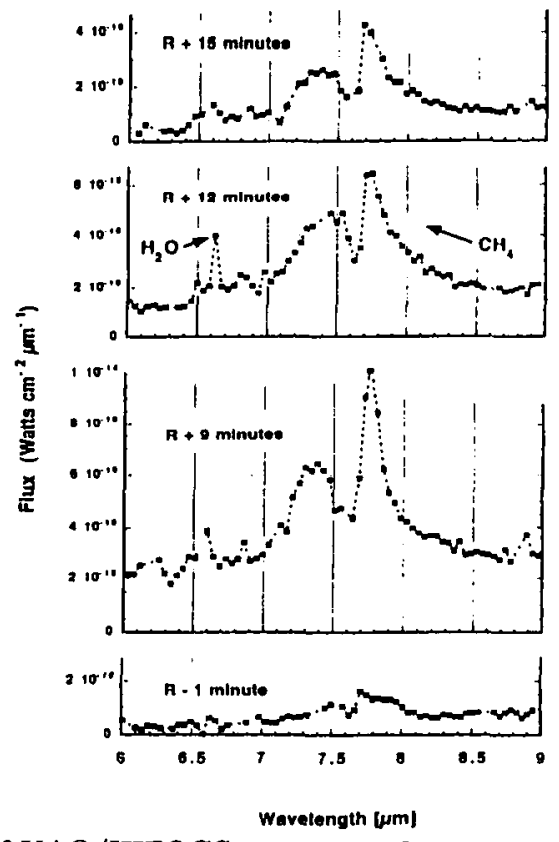

FIGURE 3. A sequence of KAO/HIFOGS spectrum of impact $R$ splashback showing the $6.6 \mu \mathrm{m} \mathrm{H} \mathrm{H}_{2} \mathrm{O}$ band and the enhanced $7-8 \mu \mathrm{m} \mathrm{CH}_{4}$ emission. From Sprague et al. (1996).

portion of the stratosphere located above a pressure level $p_{0}$ and heated to a temperature $\mathrm{T}_{0}$, and accounting for re-absorption of the radiation by cold $\mathrm{CH}_{4}$ (Bjoraker et al. 1995a, Sprague et al. 1996). Best fits were obtained for $p_{0} \sim 3 \mu$ bar (Fig. 2). From the relative intensities of the three individual water lines near $7.7 \mu \mathrm{m}, \mathrm{T}_{0}$ was inferred to be about $1000 \mathrm{~K}$. The hot methane and water column densities were found to be similar and of order $10^{18} \mathrm{~cm}^{-2}$. The corresponding water masses are of order $2 \times 10^{12} \mathrm{~g}$ for the large impactors $\mathrm{G}$ and $\mathrm{K}, 3-14 \times 10^{10} \mathrm{~g}$ for impact $\mathrm{R}$, and $2-9 \times 10^{10} \mathrm{~g}$ for impact $\mathrm{W}$. These determinations may be regarded as lower limits to the total amount of water, as the data are sensitive only to the highest $(\sim 1000 \mathrm{~K})$ temperature, although the fact that smaller masses are found for the smallest impactors suggest that a non-trivial fraction of the total water may have been actually detected.

Besides these various splashback phase measurements, Montebugnoli et al. (1995) reported the detection of water on impact site E, 52 hours after impact, from centimetric observations at $22 \mathrm{GHz}$ performed on July 19, 1994 with the 32-m antenna in Medicina, Italy. They also mentioned the tentative observation of the same line on sites A and $\mathrm{C}$ on the same day, and on site E again on September 9, 1994. The site E, July 19, line is surprisingly narrow and strong. Because of the effects of rotational smearing, the linewidth ( $\sim 40 \mathrm{kHz}$, i.e., $0.5 \mathrm{~km} \mathrm{~s}^{-1}$ FWHM) implies an emitting region less than $1^{\prime \prime}(3500 \mathrm{~km})$ in diameter. With such a diameter, absolute calibration of the antenna temperature scale implies a brightness temperature line contrast $\Delta \mathrm{T}_{B} \geq 4000 \mathrm{~K}$, which rules out LTE thermal emission as the mechanism. Cosmovici et al. (1995) suggest a maser-type emission (with radiative pumping by solar or jovian IR photons) taking place at pressures less than 1 nbar. Simple modelling suggests an emitting region $1500-\mathrm{km}$ wide, $130-300 \mathrm{~km}$ thick, a masing optical depth of -4 and an $\mathrm{H}_{2} \mathrm{O}$ column density of $10^{18} \mathrm{~cm}^{-2}$, i.e., a water mass of $5 \times 10^{11} \mathrm{~g}$. A few checks can be made on the plausibility of the detection. Maser emission of the $22 \mathrm{GHz}$ water line in jovian conditions, not to be collisionally quenched, must, in fact, take place at $\sim 10^{-11}$ bar (J. Crovisier, priv. 
comm.), i.e., above the homopause. The diffusion time at this pressure level is $\sim 10$ minutes, implying that the observed water is in diffusion equilibrium. Since this pressure level also lies within the ionosphere, it must be wondered whether water can survive ionization. Examination of ion/neutral reactions involving water suggests that the three dominant reactions are (P. Colom, priv. comm.):

$$
\begin{aligned}
& \mathrm{H}_{2} \mathrm{O}+\mathrm{H}^{+} \rightarrow \mathrm{H}_{2} \mathrm{O}^{+}+\mathrm{H} \\
& \mathrm{H}_{2} \mathrm{O}^{+}+\mathrm{H}_{2} \longrightarrow \mathrm{H}_{3} \mathrm{O}^{+}+\mathrm{H} \\
& \mathrm{H}_{3} \mathrm{O}^{+}+\mathrm{e} \longrightarrow \mathrm{H}_{2} \mathrm{O}+\mathrm{H}
\end{aligned}
$$

Equilibrium between these reactions, using typical ionospheric densities at $10^{-11}$ bar (Atreya 1986) and rate constants (Anicich and Huntress 1986), leads to concentration ratios of $\mathrm{H}_{2} \mathrm{O} / \mathrm{H}_{3} \mathrm{O}^{+} \sim 40$ and $\mathrm{H}_{2} \mathrm{O} / \mathrm{H}_{2} \mathrm{O}^{+} \sim 4000$, i.e., neutral water remains the dominant species. Finally, water appears to be also stable against photolysis. The photolytic lifetime of an isolated water molecule at Jupiter's distance is $\sim 1$ month, and this time may be increased by factors of several by shielding effects, making the observation of water days or even two months after impact plausible. In summary, we do not see any obvious physical reason that would contradict this observation, although a complete model of the pumping mechanism remains to be elaborated.

\subsection{Carbon, monoxide}

\subsubsection{Infrared observations}

Carbon monoxide has been searched for in many observations and successfully observed in both the infrared $(2.3 \mu \mathrm{m}$ and $4.7 \mu \mathrm{m})$ and the millimeter ranges.

Although far from being fully understood at the present time, the $2.3 \mu \mathrm{m}$ observations of $\mathrm{CO}$ in the plume/splashback phase are among the most spectacular results of the SL9 campaign. Spectra at $2.3 \mu \mathrm{m}$ were obtained from a variety of telescopes and instrumentation (Steward/FSPEC (Ruiz et al. 1994), AAT/IRIS (Fig. 1), UKIRT/CGS-4 (Knacke et al. 1995), Calar Alto/MAGIC (Herbst et al. 1995a), altogether covering most medium and large impacts $(C, D, G, H, K, L, R, W)$. These spectra show $C O$ emission features (generally unresolved, except in the Steward observations) belonging to the 2-0, 3-1 and 4-2 bands. These emissions, essentially concommitant with those seen for $\mathrm{H}_{2} \mathrm{O}$, appear $\sim 10$ minutes after impact time (i.e., approximately at or slightly after the IR peak flux, which might indicate that the (O-bearing) cometary material transported in the plume hits the atmosphere after the jovian material), evolve rapidly in shape and intensity, and last about 10 minutes. The CO hot bands are diagnostic of high temperatures (1500 to $5000 \mathrm{~K}$, increasing with time [Kim et al. 1995]), consistent with strong heating during the splashback phase. The AAT/IRIS observations (Crisp and Meadows 1995) also indicate that the relative intensities of the $\mathrm{CO}$ and $\mathrm{H}_{2} \mathrm{O}$ emissions vary from impact to impact. (A preliminary analysis of these data (Meadows and Crisp 1995a) suggested that $\mathrm{CO}$ was seen in absorption prior to appearing in emission, but this conclusion was apparently erroneous and caused by confusion with methane bands). Preliminary fits to the spectra suggest total CO masses of order $3 \times 10^{12} \mathrm{~g}$ for the large impactors (Knacke et al. 1995, Crisp and Meadows 1995), but this determination is very uncertain because (i) model fitting assumes no absorption of radiation by colder background atmosphere and (ii) non-LTE effects are not considered. A potentially even more serious problem may arise from the fact that the CO cooling rate from $\sim 2000 \mathrm{~K}$ is so high that any $\mathrm{CO}$ heated up to this temperature will cool off and become invisible to the observations in some seconds. Therefore, the observations might in fact sample a "production rate" rather than a total mass (D. Crisp, priv. communication). The same problem may conceivably affect the $\mathrm{H}_{2} \mathrm{O}$ measurements in the mid-infrared (see above), and further 


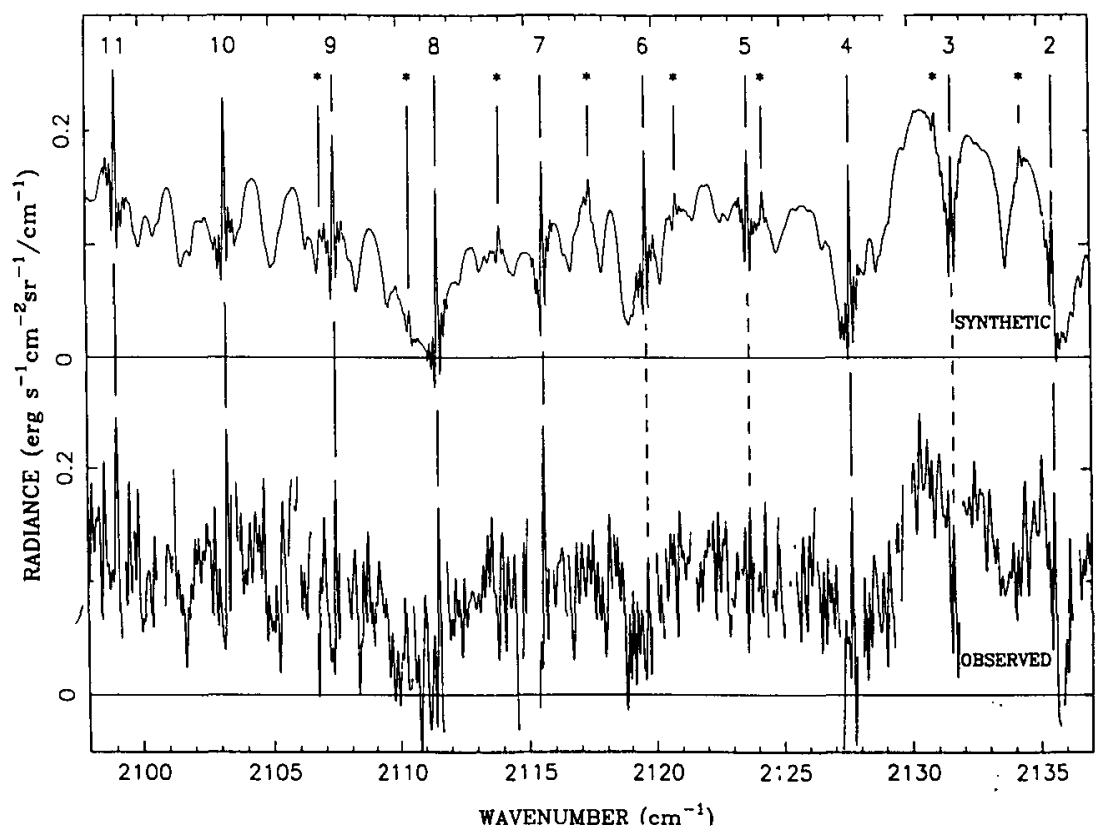

FIGURE 4. CFHT/FTS $4.7 \mu \mathrm{m}$ spectrum of site L $4.5 \mathrm{hrs}$ after impact. Resolution is $0.08 \mathrm{~cm}^{-1}$. Lines from the P-branch of the (1-0) band of ${ }^{12} \mathrm{CO}$ are marked with their rotational number. A synthetic spectrum, assuming an isothermal $\mathrm{CO}$ layer at $274 \mathrm{~K}$ (see text), is shown for comparison. The asterisks indicate the position of the ${ }^{13} \mathrm{CO}$ lines. From Maillard et al. (1995).

progress will require consistent modeling of the splashback spectra at different wavelengths.

No plume/splashback spectroscopic measurements were reported at $4.7 \mu \mathrm{m}$, and the $\mathrm{CO}$ observations at these wavelengths pertain to evolved impact sites. The most convincing detections were obtained from quasi-simultaneous observations of site $\mathrm{L}, \sim 4.5$ hours after impact, from CFHT/FTS (Maillard et al. 1995) and UKIRT/CGS4 (Brooke et, al. 1995). The high-resolution $(\mathrm{R}=25000) \mathrm{CFHT} / \mathrm{FTS}$ observations allowed the detection of 12 emission lines of the (1-0) band of ${ }^{12} \mathrm{CO}$ (superimposed on the normal tropospheric $\mathrm{CO}$ absorption features), and a marginal detection of ${ }^{13} \mathrm{CO}$ (Fig. 4). These observations in fact primarily constrain the stratospheric temperature. The ${ }^{12} \mathrm{CO}$ lines are saturated and indicate $\mathrm{T}=274 \pm 10 \mathrm{~K}$ at the level where the $\mathrm{CO}$ column density is $\sim 10^{16} \mathrm{~cm}^{-2}$. The ${ }^{13} \mathrm{CO}$ lines can be fit either (i) with a constant stratospheric temperature of $274 \mathrm{~K}$ and a CO column density of $(1.5 \pm 0.8) \times 10^{17} \mathrm{~cm}^{-2}$ (which thus appears as a lower limit to the $\mathrm{CO}$ ) or (ii) with a downward temperature decrease and a larger $\mathrm{CO}$ column density (Maillard et al. 1996) In particular, the data are fully consistent with the $\mathrm{CO}$ abundances derived from the millimeter spectra (see below). Similar results were obtained from the UKIRT/CGS4 observations, which cover only 6 lines (and at moderate spectral resolution), but for which the 1-spatial dimension allows an estimate of the size of the emission and an inference of the total CO mass (Brooke et al. 1995). These $\mathrm{CO}$ emissions were found to have disappeared one day after impact, as a result of the temperature decrease (Maillard et al. 1995).

Another $4: 7 \mu \mathrm{m}$ observation, performed from IRTF/CSHELL on August 1-2, 1994, was reported by Orton et al. (1995). A preliminary analysis suggested that some $4.7 \mu \mathrm{m}$ CO emission was still present $\sim 10$ days after the impacts, but this result was apparently caused by an instrumental artifact (K. Noll, et al. 1996). CSHELL data recorded on 
July 19 confirm that the $\mathrm{CO}$ absorption lines are back to normal 20 hours after impact (K. Noll, et al. 1996).

\subsubsection{Millimeter observations}

$\mathrm{CO}$ has been observed in many impact sites (C, E, G, H, K, L, Q1) in millimeter-wave spectroscopy. Most detections were obtained from the IRAM 30-m telescope through the CO rotational (2-1) line at $230 \mathrm{GHz}$ (Lellouch et al. 1995). Observations of this line extend from minutes to months after the impacts. The CO (1-0) line was also observed on a few occasions. The (2-1) line was also detected at the SEST-15m on July 23, on a beam position principally encompassing sites $G, Q 1$ and $R$ (BockeléeMorvan et al. 1995). Given their poor spatial resolution, all millimeter-wave measurements performed on July 21, 1994 and later include the contribution of several impact sites, which can be identified on the overall spectra from their individual velocity (see example in Bockelée-Morvan et al. 1995).

The CO rotational lines were originally detected as narrow emission lines (FWHM $2.5 \mathrm{MHz} \sim 3 \mathrm{~km} \mathrm{~s}^{-1}$ ). This linewidth implies that $\mathrm{CO}$ is located in the stratosphere, at pressures of 1 mbar or less. After July 28, they were observed in absorption (see Sec. 6.2). Preliminary modelling of the $C O(2-1)$ line on site $G, \sim 10$ hours after impact, indicated CO column densities of $\sim 1.5 \times 10^{18} \mathrm{~cm}^{-2}$, for a total CO mass of $\sim 10^{14} \mathrm{~g}$ (Lellouch et al. 1995). However, this modelling had important limitations: (i) the velocity dispersion within the site, which must contribute to the observed linewidth to some degree, was not considered (ii) a crude treatment of the geometry was adopted, with an average airmass of 2 (iii) an arbitrary thermal profile was used. This profile assumed uniform warming of the upper statosphere (at $\mathrm{p} \leq 2 \mathrm{mbar}$ ) by $30 \mathrm{~K}$ above a pre-impact Jupiter reference profile. It turns out that this assumed thermal profile is inconsistent with other data. Specifically, IRTF/IRSHELL observations of strong and weak $8 \mu \mathrm{m} \mathrm{CH}_{4}$ lines indicate that no warming larger than a few degrees occurred at pressures deeper than a few tenths of a millibar (Bézard et al. 1995a).

Improved modelling of the $\mathrm{CO}(2-1)$ line has been recently performed (Lellouch et al. in preparation). The line observed on site K, July $20, \sim 33$ hours after impact was selected, as independent information on the thermal profile and spatial extent of the emission was available from the IRTF/IRSHELL observations of the same site at $T_{i}+23$ hours. (The spatial extent of the CO was assumed to follow that of the thermal perturbation). The modelling now includes the line smearing due to velocity dispersion within the site. In addition, taking advantage of the fact that the observations were recorded during a relatively long integration time (2.5 hours), they were split into three parts depending on the site position on Jupiter's disk $\left(\phi \leq 40^{\circ} ; 40^{\circ} \leq \phi \leq 60^{\circ} ; 60^{\circ} \leq \phi \leq 80^{\circ}\right.$, where $\phi$ is the site longitude measured from the central meridian), and the three parts were separately analyzed with an improved treatment of the geometry, including variation of the secant within a site. The lines corresponding to the three parts have very different aspects and contrasts (Fig. 5), primarily a result of (i) the variation of the continuum level with the airmass and (ii) the less important contribution of the velocity smearing near the limb. Yet, all three lines of Fig. 5 can be fit with a single model, in which $\mathrm{CO}$ is confined to pressures less than $10^{-4}$ bars and its mixing ratio somewhat increases with altitude, from $2 \times 10^{-4}$ at $10^{-4}$ bars to $2 \times 10^{-3}$ at $10^{-6}$ bars. The column density is about $4 \times 10^{18} \mathrm{~cm}^{-2}$ and the total mass is $2.5 \times 10^{14} \mathrm{~g}$. Although this number is not final, as variations due to uncertainties in the thermal profile have not been considered yet (which means in particular that the conclusion that $\mathrm{CO}$ increases with altitude must not be taken too strongly at present time), it should be regarded as more reliable than that given in Lellouch et al. (1995). The combination of the lines observed in the three different 


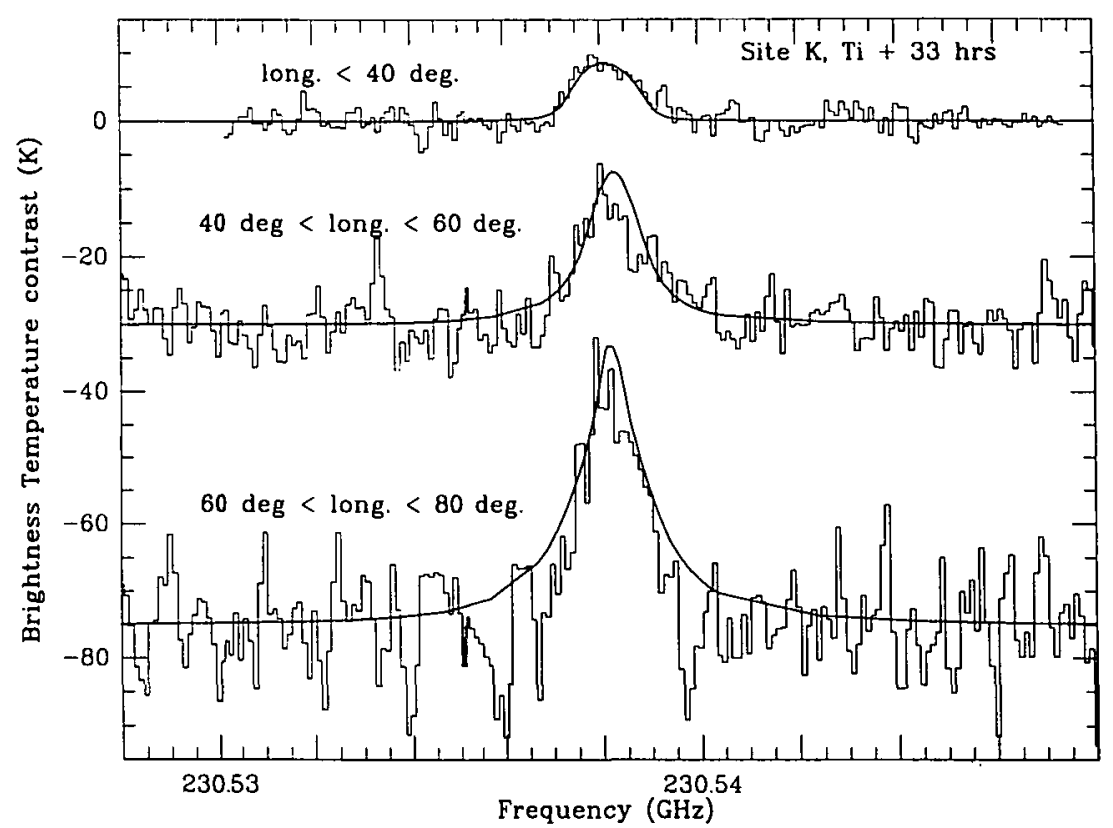

FIgURE 5. IRAM 30-m CO (2-1) spectra (histograms) of site K (July 20, UT=17:00-19:30) as a function of apparent longitude of site center with respect to central meridian. Solid lines: model fits (see text for parameters).

airmass ranges also allows us to relatively well constrain the pressure region where the $\mathrm{CO}$ is. Essentially, a significant fraction of the $\mathrm{CO}$ must be present at $\mathrm{p} \geq 10^{-5}$ bar, otherwise the synthetic line near the limb is too narrow if the line near central meridian is well fitted. Conversely, CO must also decrease very quickly at pressures $\geq 10^{-4}$ bar, otherwise the synthetic line is too broad. From this, it can be inferred that the bulk of the CO lies between $10^{-5}$ and $10^{-4}$ bar.

Millimeter-wave observations are not very well suited to the study of the plume/splashback phase because the amount of signal in the beam is severely penalized by the geometrical effect. Nevertheless, IRAM observations of impact $\mathrm{H}$ (impact time: July 18, 19:32 UT), taken at a time resolution of about $1.5 \mathrm{~min}$., seem to reveal an intriguing result. For the first 12 minutes after impact, no CO emission is seen. At 19:45 UT, a strong emission is apparently detected, which fades within 3 minutes. The timing of this emission coincides with that of the plateau in the Calar Alto lightcurve of the same impact (Herbst et al. 1995b). The striking feature is that the line is not at the limb velocity $\left(-8.8 \mathrm{~km} \mathrm{~s}^{-1}\right)$, but at an approaching velocity $\left(\sim-4 \mathrm{~km} \mathrm{~s}^{-1}\right)$ lower by $\sim 5 \mathrm{~km} \mathrm{~s}^{-1}$. Modelling of the ballistic trajectory of a plume for the initial velocity, azimuth, and elevation conditions given in Hammel et al. 1995 indicates that the velocity towards the observer remains at $\sim-11 \mathrm{~km} \mathrm{~s}^{-1}$ during the entire plume flight. The observation of a $\sim-4 \mathrm{~km} \mathrm{~s}^{-1}$ velocity is thus not understood, although it can be speculated from the timing that it might be linked to the bouncing of the plume after its impact with Jupiter's stratosphere.

\subsubsection{UV upper limit and reconciliation of measurements}

CO was not detected in the HST/GHRS spectra of site $\mathrm{G}$ and stringent upper limits on the CO column density were inferred from the absence of its fluorescence bands at 1419, 1447, 1478 and $1510 \AA$. Noll et al. (1995) find an upper limit of $6 \times 10^{14} \mathrm{~cm}^{-2}$, $\sim 5000$ times less than the millimeter value. Trafton et al. (1995) find that with the 


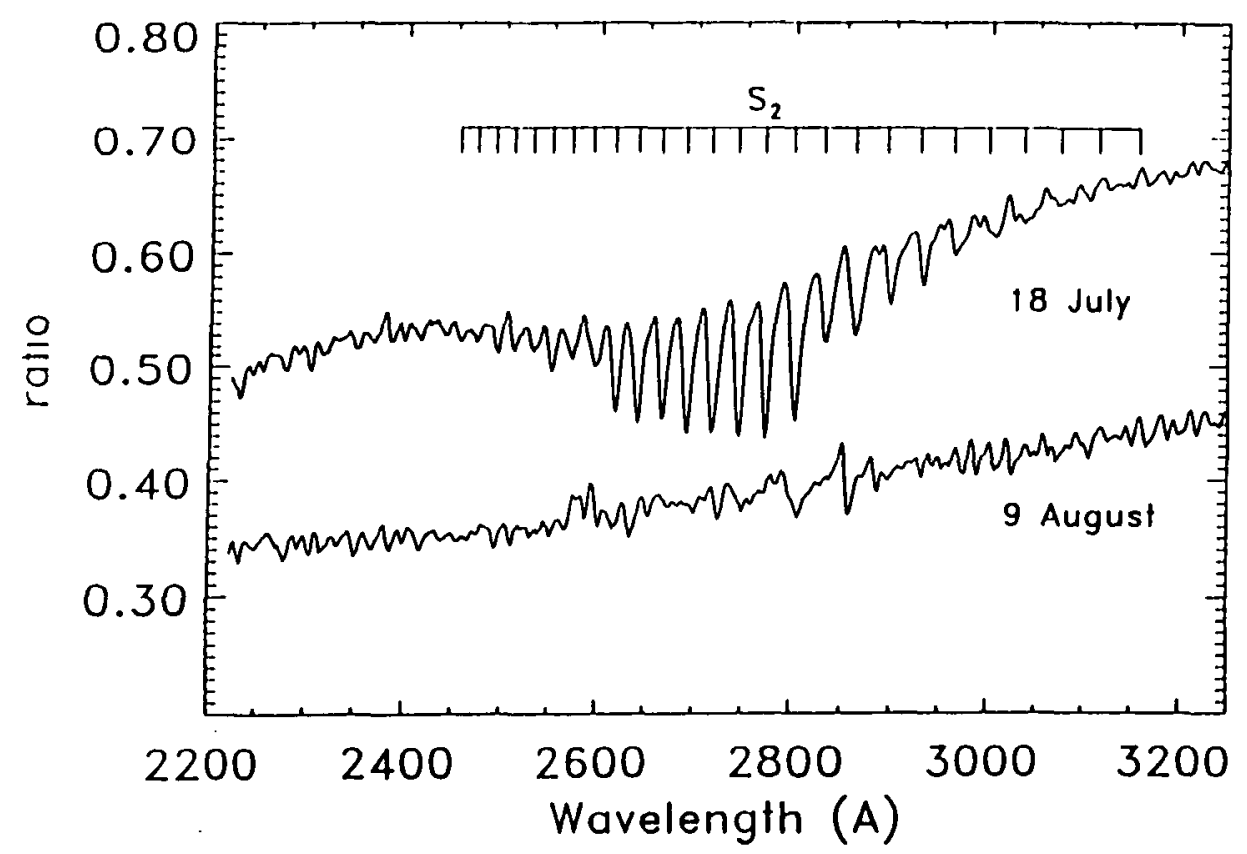

FIGURE 6. HST/FOS spectra of site G at 2200-3200 $\AA$ taken on July 18 and August 9,1994 (ratioed to a pre-impact spectrum taken on July 14) showing $\sim 16$ bands of the $\mathrm{S}_{2} \mathrm{~B}-\mathrm{X}$ system. From Noll et al. (1995).

column density given by Lellouch et al. (1995), the CO emission in the UV bands should be $\sim 8000$ Rayleigh, whereas the HST spectra indicate an upper limit of $\sim 100 \mathrm{R}$. However, both these calculations assumed optical thinness, whereas it is clear that for the relevant column densities, the individual lines of the CO UV bands are heavily saturated. Independent calculations by J. Crovisier and D. F. Strobel indicate that when saturation effects are considered, the emission is in fact photon-limited to $\sim 30 \mathrm{R}$, regardless of the $\mathrm{CO}$ abundance. These estimates are confirmed by the brightness of the Venus CO airglow (Feldman et al. 1995). There is thus no contradiction between the CO column density inferred from the millimeter and its non-detection in the UV.

As mentioned earlier, the $4.7 \mu \mathrm{m}$ observations are, in fact, more sensitive to the temperature than to the $\mathrm{CO}$ abundance, and are anyway consistent with the $\mathrm{CO}$ determinations from the millimeter. The $2.3 \mu \mathrm{m}$ spectra indicate CO masses $\sim 100$ times lower, but their analysis is fraught with complexity, and so far, it is not certain if these measurements sample the entire CO produced during the splashback. We therefore believe that the most reliable estimate of the $\mathrm{CO}$ resulting from the impact is given by the millimeter data and that a total mass of $2.5 \times 10^{14} \mathrm{~g}$ for the large impactors $(\mathrm{G}, \mathrm{K}$ ) can be provisionally adopted as a baseline value. The $\mathrm{CO}$ mixing ratio at $\mathrm{p} \leq 10^{-4}$ bar, several times $10^{-4}$, is typically $10^{5}$ times larger than the normal Jupiter CO abundance (e.g., Noll et al. (1988), implying in itself that the observed CO does not result from jovian tropospheric $\mathrm{CO}$ transported by the plume or convectively upwelling to the stratosphere.

\section{Sulfur species}

\subsection{Molecular sulfur $\left(S_{2}\right)$}

$\mathrm{S}_{2}$ was unambiguously detected in HST/FOS spectra of site G on July 18 and 21, 1994 $\left(T_{i}+3\right.$ hours and $T_{i}+3.5$ days respectively; Figs. 6 and 8$)$, through its numerous 


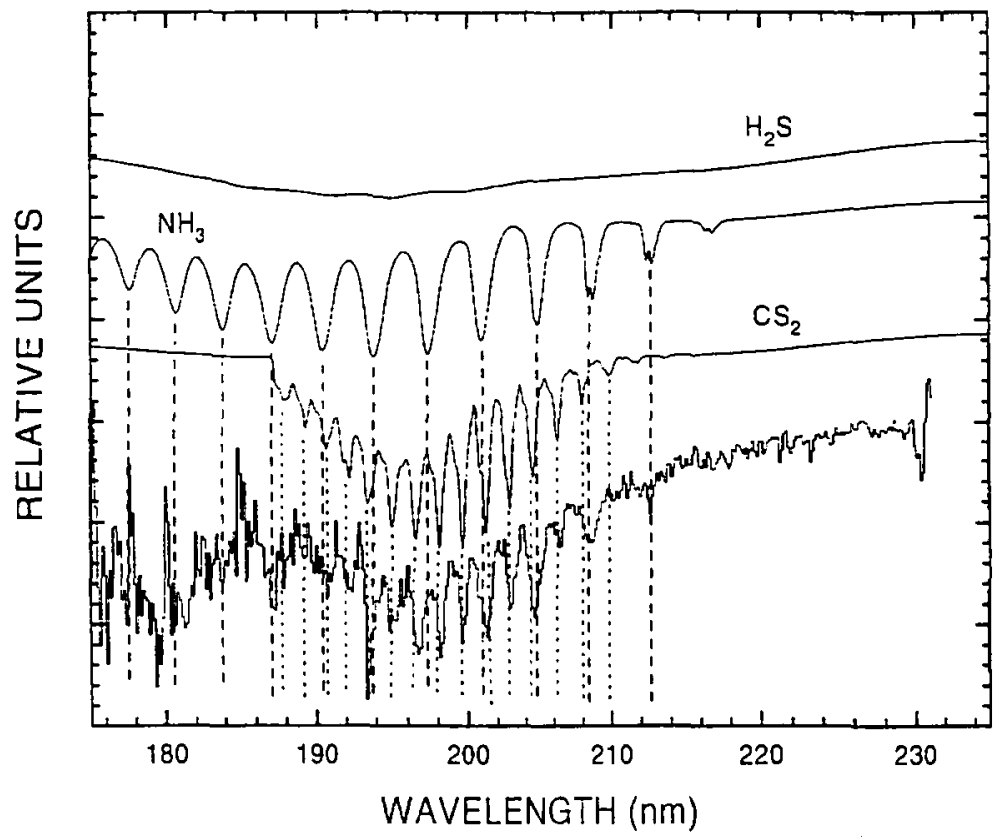

FIGURE 7. Bottom curve: HST/FOS spectrum of site G at 1750-2300 $\AA$ taken on July 18, 1994 (ratioed to a pre-impact spectrum). Top three curves. Absorption cross sections of $\mathrm{H}_{2} \mathrm{~S}, \mathrm{NH}_{3}$, and $\mathrm{CS}_{2}$ (arbitrary scale), allowing the spectral identification of $\mathrm{NH}_{3}, \mathrm{CS}_{2}$, and perhaps $\mathrm{H}_{2} \mathrm{~S}$. From Yelle and McGrath (1996).

bands belonging to the B-X system between 2500 and $3000 \AA$ (Noll et al. 1995). The bands had disappeared when HST reobserved site G, on August 9, 1994. Preliminary modelling of five of these bands (5-0 through 9-0) was performed, assuming an isothermal homogenous atmosphere at $300 \mathrm{~K}$. Matching of the equivalent widths in the July 18 spectrum indicated a column density of $3 \times 10^{18} \mathrm{~cm}^{-2}$, corresponding to a total mass of $2.5 \times 10^{13} \mathrm{~g}$ in the FOS aperture. This value was considered as a lower limit to the total $\mathrm{S}_{2}$ mass because (i) at this column density, individual $\mathrm{S}_{2}$ lines are strongly saturated, and (ii) the areal extent of $S_{2}$ is probably larger the the FOS aperture. Noll et al. (1995) proposed a baseline value of about $10^{14} \mathrm{~g}$. However, further modelling of all of the $S_{2}$ bands indicated that the observed spectrum cannot be fit at $300 \mathrm{~K}$, and that much higher temperatures $(\sim 1250 \mathrm{~K})$ are required, implying that $\mathrm{S}_{2}$ is located in Jupiter's thermosphere at $p \leq 10^{-7}$ bar (Yelle and McGrath 1995). The resulting $S_{2}$ column density is $\sim 5 \times 10^{15} \mathrm{~cm}^{-2}$, giving a mass of only $4 \times 10^{10} \mathrm{~g}$ in the FOS aperture.

\subsection{Carbon disulfide}

$\mathrm{CS}_{2}$ was detected in HST/FOS spectra of site G, on July 18, July 21, August 9, and August 23, 1994 (ratioed to a pre-impact spectrum taken on July 14) from its numerous bands between 1850 and $2100 \AA$, belonging to the ${ }^{1} \Sigma_{g}^{+}-{ }^{1} B_{2}\left({ }^{1} \Sigma_{u}^{+}\right)$system (Fig. 7). (The same spectra also show bands due to $\mathrm{NH}_{3}$ (see Sec. 4.1.1), which become progressively more prominent relative to $\mathrm{CS}_{2}$ after July 18). The emphasis of the analysis so far has been put on the July 18 spectrum. The preliminary analysis by Noll et al. (1995) indicated a $\mathrm{CS}_{2}$ column density of $0.5-2 \times 10^{15} \mathrm{~cm}^{-2}$. Further determinations relied on an improved modelling of the scattering properties of aerosols at UV wavelengths. Following West et al. (1995), Atreya et al. (1995) assume that the aerosol unit optical depth level is 
located at 0.3 to $10 \mathrm{mbar}$, and find a column density of $0.43-1.1 \times 10^{15} \mathrm{~cm}^{-2}$ at this level. Yelle and McGrath (1996) infer essentially the same number $\left(0.8 \times 10^{15} \mathrm{~cm}^{-2}\right)$ from the same spectrum, and find a decrease of the $\mathrm{CS}_{2}$ abundance with time, by factors of $2-3$ on August 9 and $\sim 5$ on August 23, compared to July 18. There is, therefore, a consensus on the $\mathrm{CS}_{2}$ abundance. In addition, Yelle and McGrath (1996) strongly emphasize that a good fit of the HST/FOS spectra can be obtained only if $\mathrm{CS}_{2}$ lies above $\mathrm{NH}_{3}$ and the bulk of the aerosols (i.e., at pressures $\leq 1 \mathrm{mbar}$ for July 18 and $\leq 0.1$ mbar for August 9 ), a condition that Atreya et al. do not require. It can be finally noted that $\mathrm{CS}_{2}$ has a very strong band at $6.5 \mu \mathrm{m}\left(\nu_{3} ; \mathrm{S}=2300 \mathrm{~cm}^{-2} \mathrm{~atm}^{-1}\right)$, which may affect the KAO/HIFOGS spectra and provide additional constraints on the $\mathrm{CS}_{2}$ distribution.

\subsection{Hydrogen sulfide}

The tentative detection of hydrogen sulfide was announced during the impact week and in the first HST spectroscopy paper (Noll et al. 1995), on the basis of the broad absorption centered at $2000 \AA$ in the HST/FOS spectra of site G. A preliminary estimate of the $\mathrm{H}_{2} \mathrm{~S}$ column density was given as $2-5 \times 10^{16} \mathrm{~cm}^{-2}$. Further investigations by the HST team lead to divergent conclusions about the presence of $\mathrm{H}_{2} \mathrm{~S}$. Yelle and McGrath (1996) note that the HST/FOS spectrum of July 18 has a positive slope at 2100-2300 $\AA$, while the reflectivity in the August 9 and 23 spectra is flat in this region. Since the $\mathrm{H}_{2} \mathrm{~S}$ cross sections exhibit a similar slope longward of $2100 \AA$, they attribute this behaviour to the presence of $\mathrm{H}_{2} \mathrm{~S}$ on July 18 and its absence on subsequent dates (Fig. 7). They also moderately favor the scenario in which $\mathrm{H}_{2} \mathrm{~S}$ is mixed with $\mathrm{NH}_{3}$ and the aerosols but below the $\mathrm{CS}_{2}$ layer. In this case, for July 18, (and assuming an aerosol imaginary index of refraction ( $k$ ) constant over the FOS range), they infer a mixing ratio of $\sim 5 \times 10^{-8}$ at $p \geq 5$ mbar. The mixing ratio is not well determined because of the uncertainties in the aerosol properties and could be 4 times smaller. Although $\mathrm{H}_{2} \mathrm{~S}$ in this model is uniformly mixed at 5-100 mbar, Yelle and McGrath (1996) recognize that the UV observations do not probe deeper than $\sim 10-20$ mbar and stress that the mixing ratio is far better constrained than the total column density ( $\mathrm{R}$. Yelle, priv. comm.). A mixing ratio of only $10^{-8}$ is inferred if $\mathrm{H}_{2} \mathrm{~S}$ is assumed uniform at $0-100$ mbar. On the other hand, Atreya et al. (1995) claim that the entire HST/FOS July 18 spectrum can be fit without invoking the presence of $\mathrm{H}_{2} \mathrm{~S}$ and obtain an upper limit on the column density of $1.2 \times 10^{16} \mathrm{~cm}^{-2}$ at the $\tau=1$ aerosol level. This corresponds to a mixing ratio $\leq 10^{-8}$ (resp. $3.5 \times 10^{-7}$ ) if this level is at 10 mbar (resp. 0.3 mbar). Finally, Caldwell et al. (1995), adopting a semi-log extrapolation of the West et al. (1995) values for the aerosol imaginary index of refraction, find that the slope at $2100-2300 \AA$ in the FOS spectrum can be matched by a uniform $\mathrm{H}_{2} \mathrm{~S}$ mixing ratio of $5 \mathrm{ppb}$, in general agreement with the Yelle and McGrath (1996) homogeneous case. However, they prefer to view this number as an upper limit, as slightly modified aerosol properties would allow a fit to the entire spectrum with no $\mathrm{H}_{2} \mathrm{~S}$ at all. Strong opinions have been expressed about the presence or absence of $\mathrm{H}_{2} \mathrm{~S}$, ranging from "the arguments for a significant abundance (of $\mathrm{H}_{2} \mathrm{~S}$ ) in the atmosphere are strong" (Yelle and McGrath 1996) to " $\mathrm{H}_{2} \mathrm{~S}$ is not detected" (Atreya et al. 1995), possibly confusing the occasional reader, and an intermediate position such as Caldwell et al. seems the most reasonable. Yelle and McGrath argue that the variation of the spectrum slope between July 18 and August 23 suggests the the absorber on July 18 was indeed $\mathrm{H}_{2} \mathrm{~S}$. However, since the $\mathrm{NH}_{3}$ and $\mathrm{CS}_{2}$ cross sections also have a slight positive slope at $210-230 \mathrm{~nm}$, this variation could be plausibly due to the established decrease of $\mathrm{CS}_{2}$ and $\mathrm{NH}_{3}$ (see Sec. 4.1.1) with time, possibly coupled with variations of aerosol properties. 


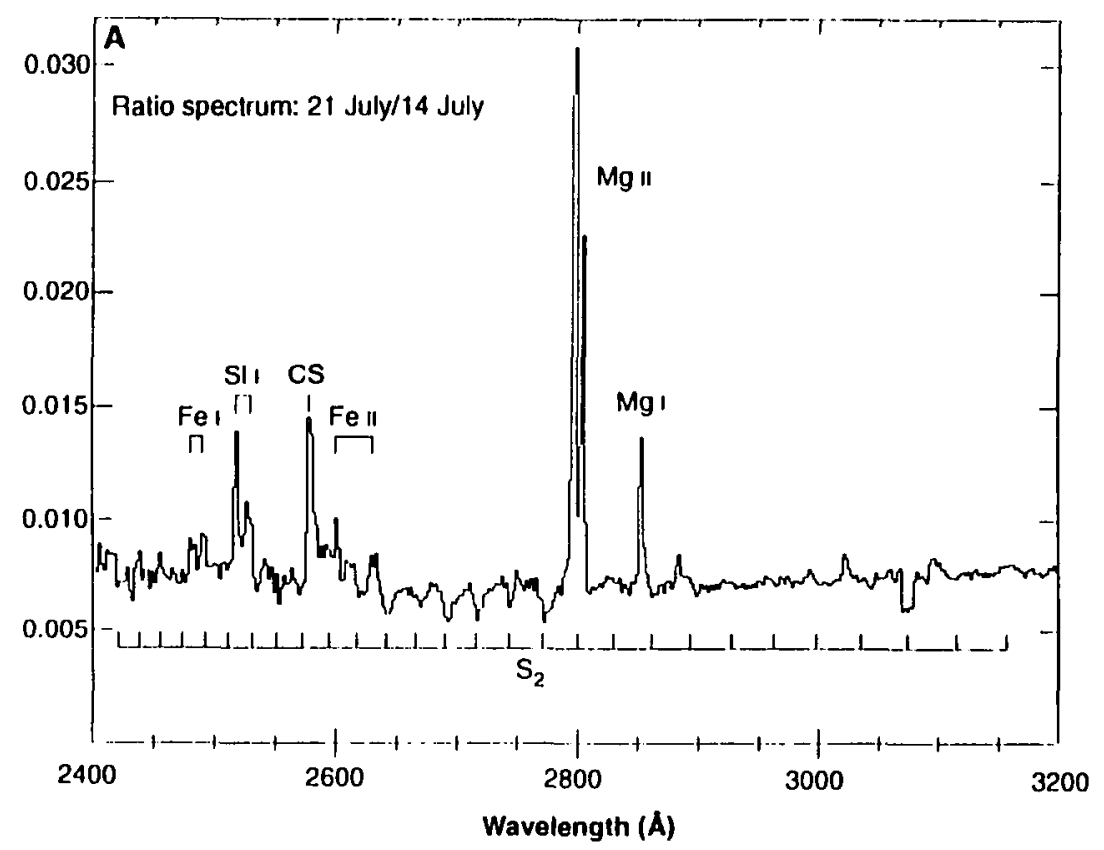

FIGURE 8. HST/FOS spectrum at 2400-3200 $\AA$ taken on July 21, 1994 (ratioed to the pre-impact spectrum). The FOS aperture encompassed site $\mathrm{G}$ and 45-min old site S. Absorption bands of $\mathrm{S}_{2}$ and emissions lines of CS and several atomic species are identified. From Noll et al. (1995).

$\mathrm{H}_{2} \mathrm{~S}$ has also been unsuccessfully searched for at millimeter (IRAM, JCMT) and infrared (IRTF/IRSHELL) wavelengths. Unfortunately, this does not seem to provide a test of the claimed UV detection. Specifically, synthetic spectra of the $\mathrm{H}_{2} \mathrm{~S} 216 \mathrm{GHz}$ line based on the $\mathrm{H}_{2} \mathrm{~S}$ distribution suggested by Yelle and McGrath (1996) are fully consistent with the non-detection of this line from IRAM observations of sites $\mathrm{G}$ and $\mathrm{H}$ on July 19 , as $\mathrm{H}_{2} \mathrm{~S}$ in this model is essentially too deep to be detectable in the millimeter range. The millimeter upper limit only constrains the $\mathrm{H}_{2} \mathrm{~S}$ at lower pressures (e.g., $\mathrm{H}_{2} \mathrm{~S} \leq 8 \times 10^{-7}$ if assumed uniform at $\mathrm{p} \leq 5 \mathrm{mbar}$ ). The upper limit from the infrared has still to be derived, but is expected to be even less stringent than from the millimeter.

\subsection{Carbon monosulfide}

CS has been observed at UV and millimeter wavelengths. In the UV, the detection comes from a limb spectrum (emission angle $79^{\circ}$ ) taken by HST/FOS on July 21 and showing the CS(0-0) transitions near $2580 \AA \hat{A}$ and a number of metallic lines (Mg I, Mg II, Si I, Fe I, Fe II, and perhaps Ni I and Ti II) (Noll et al. 1995; Carpenter et al. 1995; Fig. 8). The aperture of the instrument for this observation encompassed site $\mathrm{G}$ and 45 -min old site $\mathrm{S}$. Noll et al. (1995) assumed that the excitation mechanism for all these features was solar resonant fluorescence and estimated the $g$-factors (fluorescence efficiency factor) using a temperature of $1000 \mathrm{~K}$. In the case of CS, a column abundance of $2 \times 10^{14} \mathrm{~cm}^{-2}$ was derived, corresponding to a total mass of $9 \times 10^{9} \mathrm{~g}$ in the FOS aperture. This estimate assumes optical thinness, and therefore should be viewed as a lower limit, although reasonably accurate, according to Noll et al. It is noteworthy that CS was not detected on the spectrum taken on July $18 \sim 3.5$ hours after the $\mathrm{G}$ impact. On the other hand, weak emission features seem to appear near the position of the $\operatorname{CS}(0-0)$ transition on spectra through 23 August. 
In the millimeter range, CS was detected with the IRAM-30m telescope, first through its $\mathrm{J}=5-4$ transition at $244.9 \mathrm{GHz}$, originally seen in emission on July 21 on site $\mathrm{K}$ and complexes including sites (L, G) and (G, Q, R, S) respectively (Lellouch et, al. 1995). This line has since then been monitored on a regular basis (see Sec. 6.2). Preliminary modelling of the line observed on complex $(G, Q, R, S)$ indicated that CS is confined to pressures $\leq 0.7$ mbar with a mixing ratio (assumed uniform) of $4.5 \times 10^{-8}$, corresponding to a total mass, rescaled to site $\mathrm{G}$, of $3 \times 10^{11} \mathrm{~g}$. The CS $244.9 \mathrm{GHz}$ line appears slightly broader than the $\mathrm{CO} 230.5 \mathrm{GHz}$ line for simultaneous observations. In particular, in the most frequent case when the telescope beam contained several sites, the contribution from the different sites could be more easily separated (from their individual velocity) on the CO that on the CS lines. This larger width suggests that CS is located at deeper levels than CO. Improved modelling of the CS line, similar to that presented for CO in Sec. 2.2.2, is difficult, because the method requires that the site be well isolated in the beam. The only available case is site $\mathrm{K}$, on July 21. The model indicates a CS mixing ratio of $5 \times 10^{-8}$ at $\mathrm{p} \leq 1 \mathrm{mbar}$, i.e., a column density of $5 \times 10^{15} \mathrm{~cm}^{-2}$ and a total mass of $5 \times 10^{11} \mathrm{~g}$, but the spectrum is noisy and the fit not good.

The UV and millimeter measurements give CS column densities that differ by a factor of $\sim 20$. There are several possible reasons for this discrepancy. First, the CS emission seen by HST/FOS most likely originates in the fresh $\mathrm{S}$ site rather than from the 3-day old $\mathrm{G}$ site. (Support for this comes from the metallic emissions observed simultaneously, reminiscent of the atomic emissions at visible wavelengths that lasted for $\sim 1$ hour after impact [e.g., Roos-Serote et al. 1995, Fitzsimmons et al. 1995]). On the other hand, the millimeter measurements sample sites produced hours or days earlier. Since CS is not only produced in shock chemistry (Zahnle et al. 1995, Zahnle, this volume) but also builds up photochemically from $\mathrm{CS}_{2}$ or $\mathrm{S}_{2}$ on a $\sim 1$ day time scale (Moses et al. 1995a), it is conceivable that the largest abundance seen at millimeter wavelengths results from a difference in observing time. The second point is that the UV estimate relies on the assumption that the UV $\operatorname{CS}(0-0)$ emission is due to solar fluoresence. There is, in fact, still considerable ignorance on the mechanism responsible for the visible and UV emissions, some of which cannot be explained by resonant fluorescence (see Crovisier, this volume). Third, are optical depths effects properly estimated in the case of CS (cf. the case of $\mathrm{CO}$ above)? Finally, the CS abundance measurement from the millimeterwave observations is not yet satisfactory; an improved determination will require detailed modelling of the spectra on the $(\mathrm{G}, \mathrm{Q}, \mathrm{R}, \mathrm{S})$ complex, involving separate modelling of the contribution of the different sites.

\subsection{Carbonyl sulfide}

The detection of OCS has been reported from IRAM observations at $219 \mathrm{GHz}$ of complex (W, K) performed on July 22 (Lellouch et al. 1995). The detection is significant at the 6- $\sigma$ level for the line area. Preliminary modelling indicates a OCS mixing ratio of $2 \times 10^{-7}$ at $\mathrm{p} \leq 1 \mathrm{mbar}$. The total OCS mass, rescaled to the $\mathrm{G}$ site, is $\sim 3 \times 10^{12} \mathrm{~g}$, i.e., approximately 10 times that of CS. The inferred mixing ratio is consistent with an upper limit obtained from the HST/FOS spectrum of site G, July 18 , namely OCS $\leq 10^{-6}$ at $p$ $\leq 1$ mbar (Yelle and McGrath 1996). Nonetheless, this single detection was not confirmed by observations at other wavelengths; an attempt from IRAM on August 19, 1994, when the $\mathrm{CO}$ and $\mathrm{CS}$ were observed in absorption, was also unsuccessful. 


\section{Nitrogen species}

\subsection{Ammonia}

Unlike most other molecular species detected after the SL9 impacts (and like CO, and $\mathrm{PH}_{3}$ ), ammonia is normally observable in Jupiter's atmosphere in the ultraviolet, visible and infrared $(5$ and $10 \mu \mathrm{m}$ ) ranges. These observations have established that the vertical distribution of ammonia is governed by condensation at 300-600 mbar and photolysis at upper levels, leading to unobservable amounts above the tropopause (see e.g., Atreya 1986). UV and $10 \mu \mathrm{m}$ observations of the impact sites have revealed a profound modification of this vertical distribution, with the injection of considerable amounts of ammonia in the stratosphere.

\subsubsection{Ultraviolet observations}

Disk center ultraviolet observations of Jupiter normally detect ammonia bands at 1900$2200 \AA$ which originate from the upper troposphere $(\sim 100-300$ mbar) (e.g., Wagener et al. 1985). However, in spectra taken close to Jupiter's limb, which probe higher levels, these bands may become invisible, as exemplified by a pre-impact spectrum taken by HST/FOS on July 14 at an emission angle of $73^{\circ}$, and which only shows spectral signatures of acetylene (Yelle and McGrath 1996). HST/FOS observations of impact site G at 1650-2300 $\AA$ were taken on July 18, July 21, August 9 and August 23, at emission angles of $54^{\circ}, 73^{\circ}, 79^{\circ}$ and $67^{\circ}$ (Noll et al. 1995). Ratioing these spectra to the preimpact spectrum of July 14 makes the ammonia bands at 1900-2200 $\AA$ clearly appear and indicates the presence of $\mathrm{NH}_{3}$ in the stratosphere (Fig. 7). As for $\mathrm{CS}_{2}$, and using the same approach, most of the analyses have been dedicated to the July 18 spectrum. Noll et al. (1995) infer a vertical column density of $\sim 10^{16} \mathrm{~cm}^{-2}$, within a factor of 2 . Atreya et al. (1995) obtain a similar number $\left(0.25-1.3 \times 10^{16} \mathrm{~cm}^{-2}\right)$ at the $\tau=1$ aerosol level, assumed to lie at $0.3-10 \mathrm{mbar}$. If this level is located at $1 \mathrm{mbar}$, this corresponds to a mixing ratio of $0.2-1 \times 10^{-7}$. Yelle and McGrath (1996) find that $\mathrm{NH}_{3}$ is confined to pressures $\geq 5$ mbar and determine a mixing ratio of $10^{-7}$ rather than a column density. As for $\mathrm{H}_{2} \mathrm{~S}$, they mention that the $\mathrm{NH}_{3}$ mixing ratio could be 4 times smaller. Yelle and McGrath further analyze the August 9 and 23 spectra for which they find ammonia mixing ratios of $10^{-7}$ and $3 \times 10^{-8}$, respectively. This decrease is consistent with a photchemical destruction on a month timescale, although the HST/FOS observations only sample the ammonia in the dense core region, so that part of the effect may be due to horizontal spreading.

\subsubsection{Ten-micron observations}

Most of the infrared observations of ammonia in the impact sites was obtained at $10 \mathrm{mi}$ crons. In normal (pre-impact) conditions, ammonia $10 \mu \mathrm{m}$ lines are formed in Jupiter's troposphere and thus appear as broad absorption features (e.g., Kunde et al. 1982). Observations of impact sites detected emission cores in the center of these absorptions, demonstrating the presence of ammonia in Jupiter's stratosphere. The most detailed results were obtained from the IRTF/IRSHELL spectro-imaging observations of site $\mathrm{K}$ 23 hours, 5 days and 10 days after impact (Griffith et al. 1996). These measurements have a spectral resolution of 15000 and a spatial resolution of $1.4^{\prime \prime}(\sim 5000 \mathrm{~km})$, so that they provide vertical, spatial and temporal information on the ammonia distribution (Fig. 9). In addition, thermal profile information is retrieved from quasi-simultaneous observations of methane lines near $1234 \mathrm{~cm}^{-1}$. The site $\mathrm{K}$ observations at 908 and $948 \mathrm{~cm}^{-1}, 23$ hours after impact, indicate that $\mathrm{NH}_{3}$ is mostly located at $\mathrm{p} \leq 10$ mbar. For the pixel of maximum emission, the column density is $2-5 \times 10^{17} \mathrm{~cm}^{-2}$ above 40 mbar. 


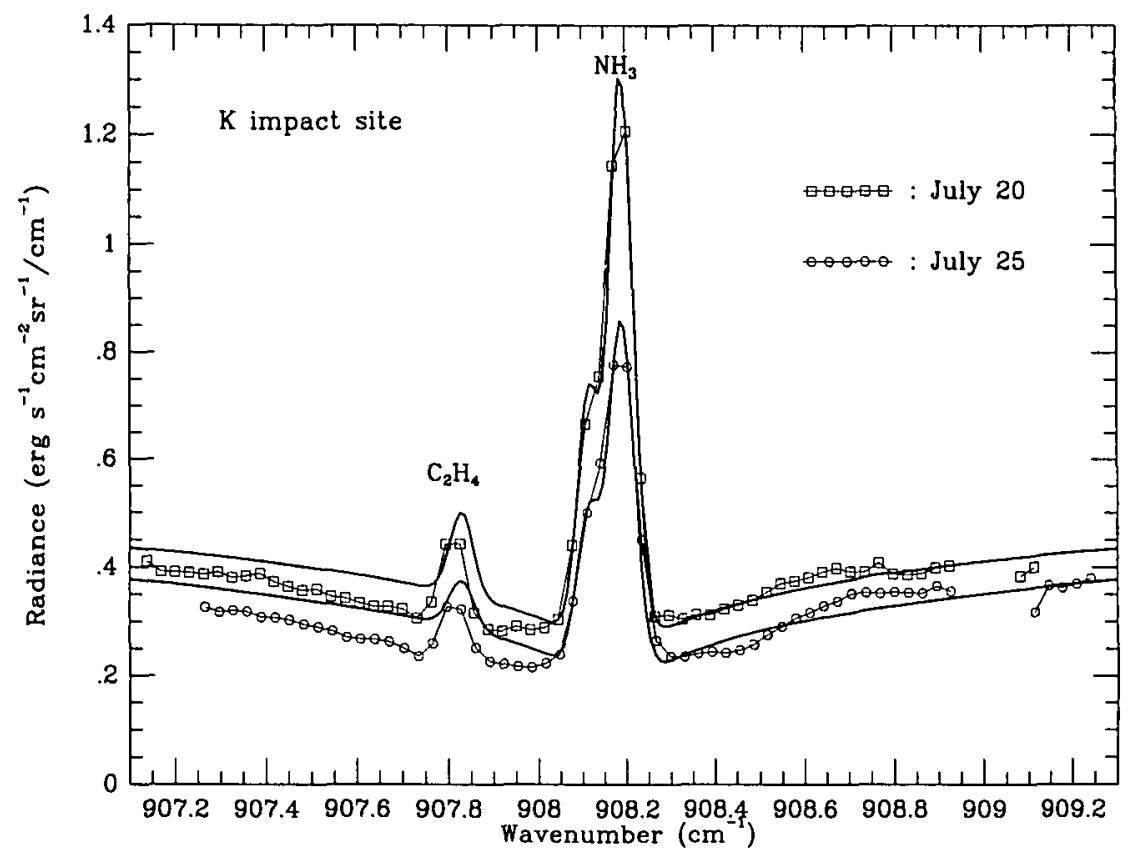

FIgURE 9. IRTF/IRSHELL spectra near $908 \mathrm{~cm}^{-1}$ of site $\mathrm{K}, 23$ hours and 10 days after impact, showing stratospheric emission from $\mathrm{NH}_{3}$ and $\mathrm{C}_{2} \mathrm{H}_{4}$. Note the decrease in time of the $\mathrm{NH}_{3}$ emission. Adapted from Griffith et al. (1996) (courtesy C. Griffith, B. Bézard).

The mass of ammonia integrated over the $\mathrm{K}$ site is $\sim 10^{13} \mathrm{~g}$. Observations 5 and 10 days after impact indicate that $\mathrm{NH}_{3}$ spreads horizontally with time. Its vertical distribution is depleted with time as a function of altitude. Specifically, the column density at the central pixel decreases by $60 \%$ over 10 days, but given the lateral spreading, the total mass over the site decreases by only $25 \%$, in excellent agreement with predictions from photochemical modelling (Moses et al. 1995b). Similar observations were obtained with the Palomar/SpectroCam 10 spectro-imager (Conrath et al. 1995). The calibration of these data is still preliminary, but first results suggest a stratospheric mixing ratio of $2 \times 10^{-7}$, roughly consistent with the IRTF/IRSHELL results.

Ammonia $10 \mu \mathrm{m}$ emission was also detected in heterodyne observations performed at Mt. Wilson/ISI telescope (Betz et al. 1995) and at the IRTF (IRHS spectrometer; Fast et al. 1995a). Observations at Mt. Wilson were recorded between July 16 and August 8, 1994. Narrow line emission was observed on impact sites E, G, H, K, L and Q1, and upper limits were obtained on sites $\mathrm{A}$ and $\mathrm{C}$ and on regions adjacent to the large sites. The strongest emission was seen on July 21 from site $G, \sim 3$ days after impact, where the aQ(2,2), aQ $(6,6)$ and $\mathrm{aQ}(9,8)$ lines of the $\nu_{2}$ band were detected (Fig. 10). The first two lines appear to be saturated and constrain the stratospheric temperature to be $\sim 200 \mathrm{~K}$, while the third one is weaker and provides a preliminary estimate of the column density of $1.5-2.0 \times 10^{17} \mathrm{~cm}^{-2}$. The linewidths $\left(\sim 1.7 \mathrm{~km} \mathrm{~s}^{-1}\right.$ FWHM at the beginning of the observing period and $2-3 \mathrm{~km} \mathrm{~s}^{-1}$ towards the end) are mostly due to velocity dispersion within the sites, and from the absence of Lorentzian linewings, Betz et, al. (1995) conclude that ammonia is primarily seen at $\mathrm{p} \leq 2-3 \mathrm{mbar}$. At deeper levels, $\mathrm{NH}_{3}$ is either absent or at a temperature below the detection threshold of the experiment $(\sim 165 \mathrm{~K})$.

Similar data were obtained from IRTF/IRHS measurements of sites K, Q1 and R on July 23, 26 and 29. From a lineshape analysis, including deconvolution of the rotational 


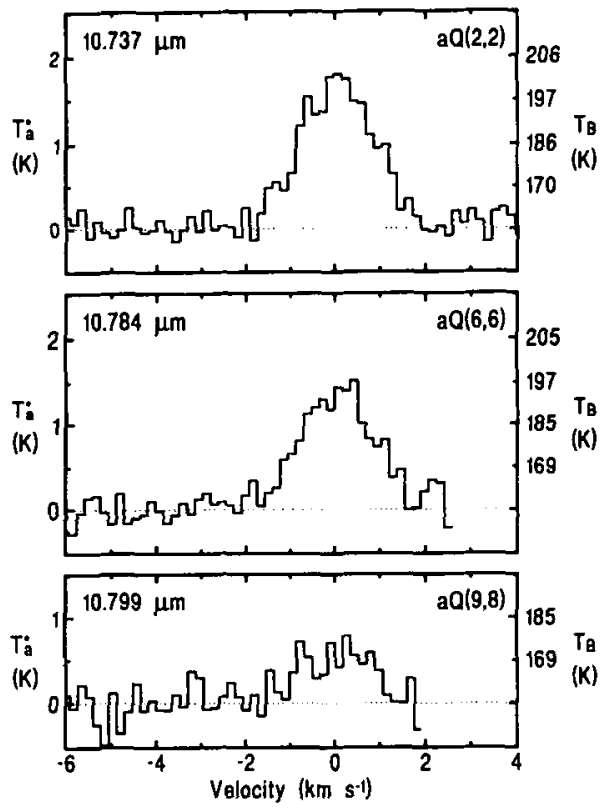

FigurE 10. Mt. Wilson/ISI heterodyne observations of $3 \mathrm{NH}_{3}$ emission lines on site G, July 21, 1994. From Betz et, al. (1995).

broadening, Fast et al. (1995a) find that Lorentz broadening is visible in their data and indicates that $\mathrm{NH}_{3}$ is mostly present at 1-10 mbar. For sites $\mathrm{Q} 1$ and $\mathrm{R}, 8$ days after impact, assuming uniform mixing in the stratosphere, they determine mixing ratios of $8 \times 10^{-9}$ and $4 \times 10^{-9}$ corresponding to column densities of $3 \times 10^{16}$ and $1.5 \times 10^{16} \mathrm{~cm}^{-2}$, respectively. Kostiuk et al. (1995) find that the best fit of the Q1 data is obtained for $\mathrm{NH}_{3}=2 \times 10^{-8}$ at $\mathrm{p} \leq 10$ mbar, giving a column density of $\sim 2 \times 10^{16} \mathrm{~cm}^{-2}$.

Finally, broad-band IRTF/MIRAC2 images show enhanced $\mathrm{NH}_{3}$ emission over nearly every impact site (Orton et al. 1995). This emission appears to decay from July 17 to August 6, consistent with photochemical destruction.

\subsubsection{Ammonia in the plume/splashback}

The AAT/IRIS spectra of the plume/splashback phase of many impacts, in which $\mathrm{H}_{2} \mathrm{O}$ and $\mathrm{CO}$ were unambiguously detected (Meadows and Crisp 1995a), also contain a relatively clear signature of ammonia at $2.04 \mu \mathrm{m}$ (Fig. 1). As for the other species, the associated abundance remains to be determined. If real, this observation is very important because it shows that some $\mathrm{NH}_{3}$ is dragged or formed in the plume, whereas all previous observations see ammonia in the mid-stratosphere (1-50 mbar), which presumably results from slower vertical mixing following the impacts. In addition, the ammonia emission at $2.04 \mu \mathrm{m}$ appears earlier than the $\mathrm{CO}$ and $\mathrm{H}_{2} \mathrm{O}$ emissions seen in the same spectra (Sec. 2), suggesting that $\mathrm{NH}_{3}$ is ejected at lower energies and to lower altitudes than $\mathrm{CO}$ and $\mathrm{H}_{2} \mathrm{O}$ (Meadows and Crisp 1995b). If ammonia is detected at $2.04 \mu \mathrm{m}$, one might expect to see it also in the stronger $10 \mu \mathrm{m}$ band observed during the splashback phase of impacts $\mathrm{R}$ and $\mathrm{W}$ by KAO/HIFOGS. This issue, however, has not been investigated yet (D. Hunten, priv. comm.).

\subsubsection{Reconciliation of the site measurements}

Intercomparison of the different ammonia site measurements shows no big contradiction, although some differences can be noted. The IRTF/IRSHELL column density of 2-5 
$\times 10^{17} \mathrm{~cm}^{-2}$ above 40 mbar, measured one day after the K impact (Griffith et al. 1996), is largely consistent (1-3 times larger) with that determined by Betz et al. (1995) on site G at $\mathrm{T}_{i}+3$ days, but $\sim 10$ times larger than the value obtained by Fast et al. (1995a). Comparison with the HST/UV determinations is difficult because the level probed by these measurements is unknown. If ammonia is assumed to extend down to $40 \mathrm{mbar}$, the average mixing ratio determined by Yelle and McGrath (1995) $\left(10^{-7}\right)$ corresponds to a column density of $5 \times 10^{17} \mathrm{~cm}^{-2}$, consistent with IRSHELL. Clearing out the residual differences between the various measurements will require detailed comparisons, including fitting of the different datasets with a single model. One point that can be made is that different measurements may see different abundances, in part because ammonia varies with time. This argument is true but should not be pushed too much because both the IRSHELL and the UV measurements show that the ammonia column at the site center decreases by a factor of $\sim 2$ only over a week or so. Specifically, the $\mathrm{NH}_{3}$ column on site $\mathrm{K}, 10$ days after impact inferred by Griffith et al., is still $\sim 6$ times larger than the value obtained by Fast et al. on site Q1, 8 days after impact. The second aspect is that the 10 micron measurements depend on the assumed thermal profile. In this respect, the IRSHELL determination (Griffith et al. 1996) seems to be the most reliable, because it makes use of a thermal profile simultaneously determined from methane or acetylene lines. For example, the thermal profile used by Fast et. al. (1995a), 8 days after impact, is several degrees warmer than indicated by IRSHELL measurements at $T_{i}+10$ days (B. Bézard, priv. comm.).

A key parameter for understanding the origin of ammonia is its vertical distribution. In this respect, the situation is not completely clear. Griffith et, al. find that most of the ammonia is present at $\mathrm{p} \leq 10 \mathrm{mbar}$, but cannot exclude that it be entirely located at much lower pressures. The two heterodyne measurements give diverging results. Unlike Betz et al., Fast et al. find evidence for pressure broadening in their data, implying that ammonia is relatively deep (1-10 mbar). The reason for this discrepancy is obscure; it might in part be due to a vertical diffusion of $\mathrm{NH}_{3}$ with time, or to the fact that the highest sensitivity of the IRTF/IRHS measurements of Fast et al. allows them to probe colder (i.e., deeper) levels. The presence of $\mathrm{NH}_{3}$ at pressures $\geq 5 \mathrm{mbar}$ is inferred independently by Yelle and McGrath (1996), who, from the HST/FOS observations, essentially find that ammonia must be mixed with aerosols. Atreya et al. (1995), on the other hand, do not believe that height distribution can be extracted from these spectra (S. Atreya, priv. comm.). They mention that since the observations do not probe below levels where the aerosol optical depth exceeds the value of several, all that can be determined is the column density down to the $\tau=1$ level. Apparently, the key questions regarding the analysis of the UV spectra are: (i) at what pressure level does the $\tau=1$ aerosol level lie? and (ii) are the observations more sensitive to a mixing ratio or to an integrated column density? In favor of the conclusion that a large fraction of the ammonia is indeed mixed with the aerosols, is the fact that the Griffith et, al. column density of $\mathrm{NH}_{3}$ is $\sim 50$ times larger than that inferred by Atreya et al. (1995) at the $\tau=1$ aerosol level. In summary, there are several indications for the presence of ammonia at deeper levels than other compounds (e.g., CO), but it seems prudent to wait until the discrepancies mentioned above are solved to accept this result as definitive.

\subsection{Hydrogen cyanide}

Prior to the SL9 impacts, the detection of hydrogen cyanide on Jupiter had been announced by Tokunaga et al. (1981) from $13-\mu \mathrm{m}$ spectroscopy. A mixing ratio of $\sim 2 \times 10^{-9}$ had been inferred. Further searches for HCN in the same and other spectral 


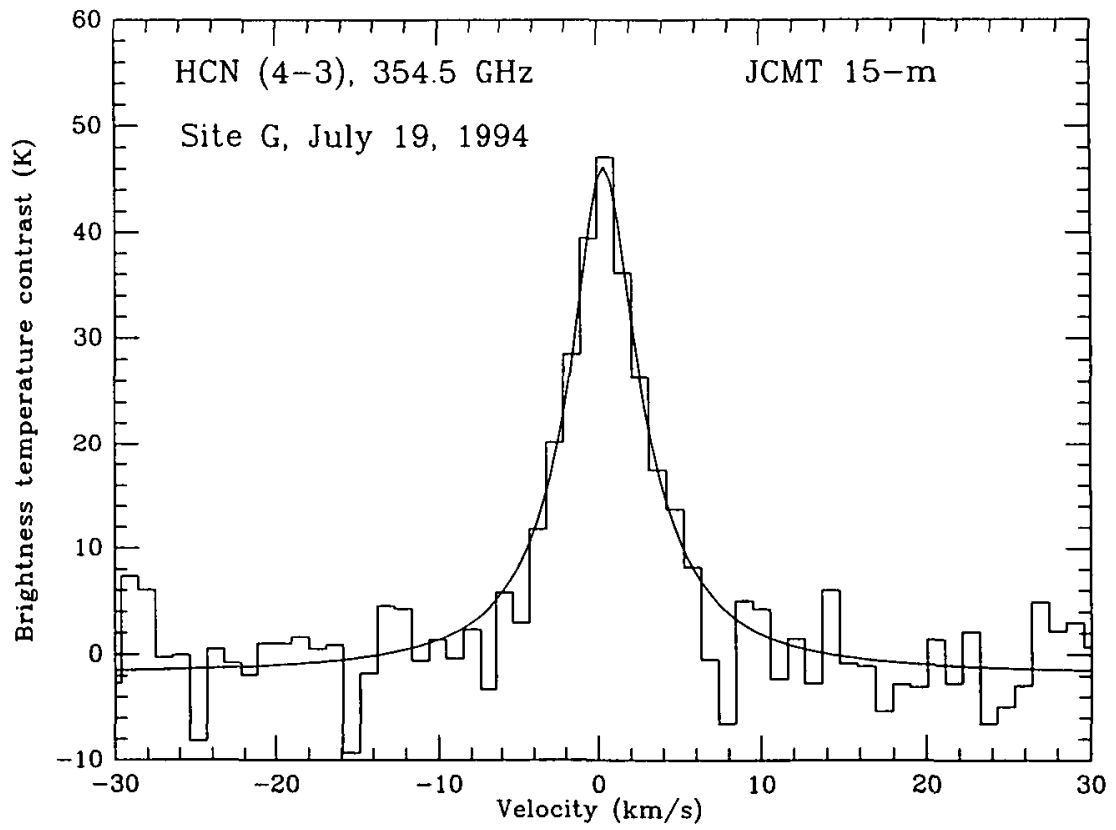

Figure 11. HCN (4-3) rotational line, observed on site G, July 19 from JCMT heterodyne observations (histograms). The model fit corresponds to a uniform HCN mixing ratio of $5 \times 10^{-8}$ at $\mathbf{p} \leq 0.5 \mathrm{mbar}$. The inset shows the associated thermal profile. This solution is non-unique, however (see text). From Marten et al. (1995).

ranges did not confirm this detection, however, and from a reassessment of the Tokunaga et al. observations, Bézard et al. (1995a) concluded that this detection was incorrect.

Following the cometary impacts, $\mathrm{HCN}$ has been observed in the millimeter/submillimeter range and at $13 \mu \mathrm{m}$. Observations at the JCMT have detected the HCN J $=4-3$ line at $354.5 \mathrm{GHz}$ on sites $\mathrm{A}, \mathrm{C}, \mathrm{F}, \mathrm{G}, \mathrm{H}$ and $\mathrm{R}$, hours and days after impact, and the $\mathrm{J}=$ 3-2 line at $2669 \mathrm{GHz}$ on site R (Marten et al. 1995; Fig. 11). As for CO and CS, these lines were originally detected as narrow emission features, but had turned to absorption when they were reobserved in September 1994 and later. Preliminary modelling of this line was accomplished using a model similar to that of Lellouch et al. (1995) for CO, i.e., using a priori assumptions on the thermal profile and a simplified geometrical treatment, and neglecting any velocity broadening within the sites. From such modelling, applied to $\mathrm{J}=4-3$ line observed on site $\mathrm{G}$, July 19 , a HCN mixing ratio of $5 \times 10^{-8}$ at $\mathrm{p} \leq 0.5 \mathrm{mbar}$ was found, i.e., a column density of $3 \times 10^{15} \mathrm{~cm}^{-2}$ (Marten et al. 1995), which corresponds to a $\sim 3 \times 10^{11} \mathrm{~g}$ mass of $\mathrm{HCN}$ if a $3^{\prime \prime}(11500 \mathrm{~km})$ site diameter is assumed. However, this solution is non-unique, as the thermal profile is unconstrained. For example, a virtually identical fit of this line can also been obtained with another thermal profile, and a gas distribution in which $\mathrm{HCN}$ is restricted to $\mathrm{p} \leq 0.1 \mathrm{mbar}$ with a $10^{-6}$ mixing ratio, giving a $10^{16} \mathrm{~cm}^{-2}$ column density. Examination of the thermal profiles in both cases show that they are in fact inconsistent with other data (they are too warm in the 0.1-1 mbar region). Nevertheless, these two solutions, which differ in the total HCN mass by a factor of $\sim 3$, give an idea of the precision that can be expected from the analysis of millimeter-wave data alone.

HCN was also successfully detected at $744.5 \mathrm{~cm}^{-1}$ in IRSHELL/IRTF observations of several impact sites (A, E, H on July $20, G, L, K+W$ on July 30 and $G+Q+R$ on July 31). As for $\mathrm{NH}_{3}$, the $\mathrm{HCN}$ emission was imaged with a $1.5^{\prime \prime}$ resolution (Bézard 
et al. 1996). Thermal profile information was obtained from $\mathrm{CH}_{4}$ or $\mathrm{C}_{2} \mathrm{H}_{2}$ emission. For site $\mathrm{E}$ on July 20, 2.6 days after impact, a HCN column density of $0.9 \pm 0.2 \times 10^{16} \mathrm{~cm}^{-2}$ is inferred. The $\mathrm{HCN}$ mixing ratio is $\sim 7 \times 10^{-7}$ at $\mathrm{p} \leq 0.1$ mbar (assuming uniform mixing above this level), and the total mass over site $\mathrm{E}$ is $6 \times 10^{11} \mathrm{~g}$. For site $\mathrm{K}, 10$ days after impact, the $\mathrm{HCN}$ mass is $7 \times 10^{11} \mathrm{~g}$.

Infrared and millimeter/submillimeter observations thus appear to give consistent results on the location and total mass of HCN. It must be emphasized that the analysis of the JCMT observations is still preliminary, and that further modelling, similar to that presented for $\mathrm{CO}$ in Sec. 2.2.2, is required.

\section{Phosphine, hydrocarbons and upper limits}

\subsection{Phosphine}

A tentative detection of $\mathrm{PH}_{3}$ has been reported from Palomar/SpectroCam 10 observations of site $\mathrm{L}$ at $10.05 \mu \mathrm{m}$ (Conrath et al. 1995). As compared to a spectrum taken on an adjacent impact-free region, the spectrum of site $\mathrm{L}$ at $987-1002 \mathrm{~cm}^{-1}$ shows three distinctive features: (i) an increased continuum level (by about $2 \mathrm{~K}$ ) throughout the spectral range, probably due to dust (silicate) emission (Conrath, this volume; West, this volume), (ii) a strong emission line due to $\mathrm{NH}_{3}$ near $993 \mathrm{~cm}^{-1}$, (iii) extra emission, near $991.5 \mathrm{~cm}^{-1}$, tentatively attributed to the $\mathrm{Q}$ branch of the $\nu_{2}$ band of $\mathrm{PH}_{3}$. A preliminary fit of this emission suggests a phosphine mixing ratio of $2 \times 10^{-8}$, assuming uniform mixing in the stratosphere. This abundance should conservatively be regarded as an upper limit (B. Conrath, priv. comm.). A more stringent upper limit can, in fact, be derived from the HST/FOS spectrum of site G. These data indicate a maximum $\mathrm{PH}_{3}$ column density of $3.3 \times 10^{14} \mathrm{~cm}^{-2}$ at the aerosol unit optical depth level (Atreya et al. 1995). Assuming this level is at $1 \mathrm{mbar}$, the $\mathrm{PH}_{3}$ mixing ratio is $\leq 3 \times 10^{-9}$.

\subsection{Hydrocarbons}

\subsubsection{Ethylene}

Emission from $\mathrm{C}_{2} \mathrm{H}_{4}$ has been detected at $907.5 \mathrm{~cm}^{-1}$ in IRTF/IRSHELL spectra of site K (Griffith et al. 1996; see Fig. 9). Like for $\mathrm{NH}_{3}$, this emission was observed 23 hours, 5 days and 10 days after impact. Orton et al. (1995) gave a rough estimate of $3 \mathrm{ppb}$ for the $\mathrm{C}_{2} \mathrm{H}_{4}$ mixing ratio, assumed to be uniform in the stratosphere. Further analysis of the July spectrum ( $T_{i}+23$ hours), making use of line strengths measured for that purpose, indicated that the ethylene emission could be fit with a distribution similar to HCN (i.e., restricted to $\mathrm{p} \leq 0.1 \mathrm{mbar}$ ) and a total mass of $\sim 3 \times 10^{12} \mathrm{~g}$ (Griffith et al. 1996). Although ethylene had been detected previously in Jupiter's stratosphere (in auroral regions (Kim et al. 1985) and subsequently at equatorial latitudes [Kostiuk et al. 1993]), its "normal" mixing ratio is of order $10^{-9}$ only. The observation of a $\sim 1000-5000$ times enhanced $\mathrm{C}_{2} \mathrm{H}_{4}$ abundance implies formation from shock chemistry, as is the case for $\mathrm{CO}$ (see above).

\subsubsection{Other hydrocarbons}

Observational evidence for a variation in the distribution and abundance of other hydrocarbons is not clear. As mentioned several times in this review, considerable enhancements of the emissions of $\mathrm{CH}_{4}$ at $8 \mu \mathrm{m}$ and $\mathrm{C}_{2} \mathrm{H}_{2}$ at $13.7 \mu \mathrm{m}$ have been observed, but they can be attributed to temperature effects. Entry and fallback models (Zahnle, this volume) predict that the amount of methane, acetylene and ethane produced from shock chemistry is a small fraction (percent or less) of the material preexisting in Jupiter's 


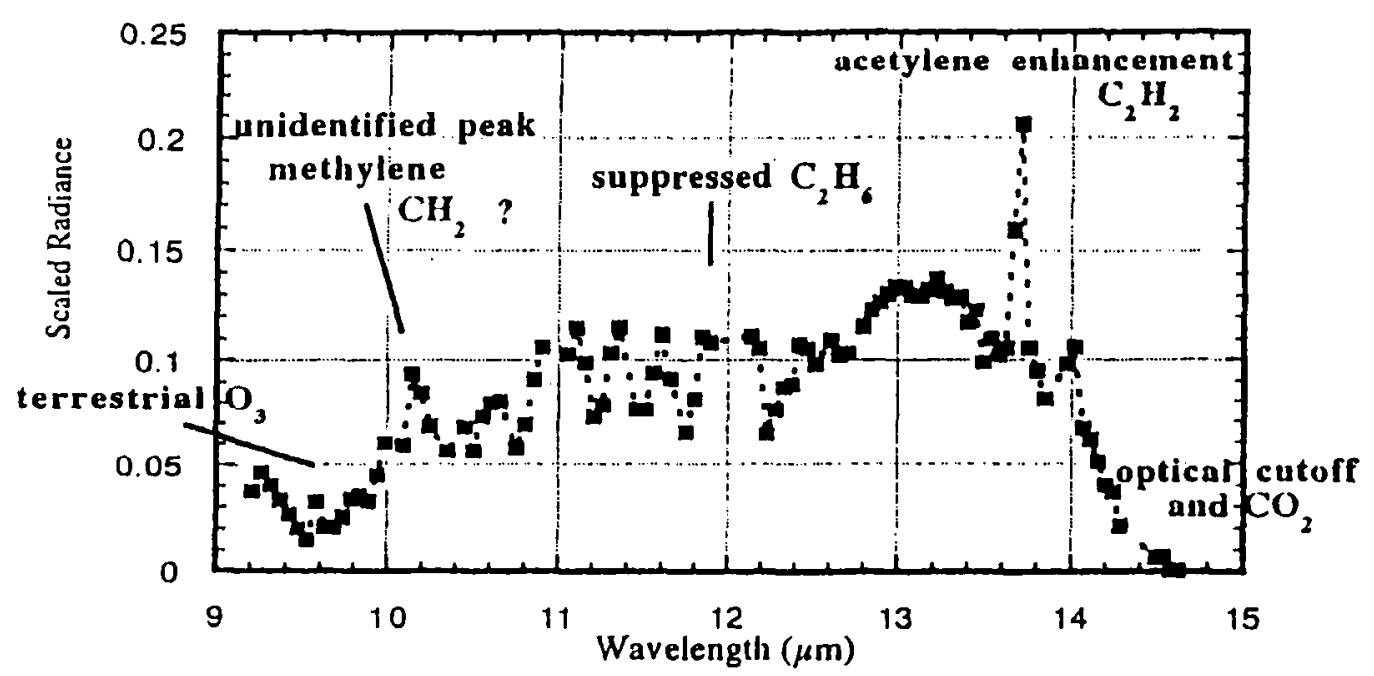

FIGURE 12. KAO/HIFOGS spectrum of fragment $R$ splashback at 9-14 microns. A spectrum from the Northern hemisphere has been subtracted and the telluric absorptions have not been fully corrected. Enhanced acetylene and depressed ethane emission are seen, along with at least one unidentified peak. Courtesy A. Sprague.

stratosphere. If true, this fully justifies the use of the IR emissions as thermometers of the jovian stratosphere, but it makes searches for variations of $\mathrm{C}_{2} \mathrm{H}_{2}$ and $\mathrm{C}_{2} \mathrm{H}_{6}$ at other wavelengths hopeless. In the UV, in contrast to the pre-impact spectrum of July 14, the HST/FOS July 18, site G, spectrum does not show the acetylene bands that are masked by the ammonia bands. From these data, upper limits on the $\mathrm{C}_{2} \mathrm{H}_{2}$ mixing ratio of $3 \times 10^{-7}$ and $10^{-6}$ have been inferred by Yelle and McGrath (1996) and Caldwell et al. (1995) respectively, several times larger than the normal jovian value (see review of the measurements in Gladstone et al. [1996]).

Variations of the $\mathrm{C}_{2} \mathrm{H}_{6} \nu_{9}$ band contrast at $820 \mathrm{~cm}^{-1}$ have also been observed, and appear to be un- or even anti-correlated with that of the $\mathrm{C}_{2} \mathrm{H}_{2} 13.7 \mu \mathrm{m}$ emission. For example, a KAO/HIFOGS spectrum of the $\mathrm{R}$ impact region shows strongly enhanced acetylene region, yet a suppressed $\mathrm{C}_{2} \mathrm{H}_{2}$ band (Sprague et al. 1996; Fig. 12). This does not necessarily imply a true decrease of the $\mathrm{C}_{2} \mathrm{H}_{6}$ abundance, however. The ethane emission probes deeper levels than the acetylene emission does (typically 1 mbar vs. $20 \mu$ bar; Drossart et al. 1993, Livengood et al. 1993), therefore it may be unaffected by a temperature increase at $\mathrm{p} \leq 0.1 \mathrm{mbar}$; and the apparent disappearance of the ethane band may plausibly be due to an increase of the continuum due to enlanced dust opacity rather than to a decrease of the $\mathrm{C}_{2} \mathrm{H}_{6}$ mixing ratio. The same KAO/HIFOGS spectrum show a number of new peaks between 9 and $15 \mu \mathrm{m}$, that are not identified as yet, although one of them (at $10.2 \mu \mathrm{m}$ ) might be due to methylene $\left(\mathrm{CH}_{2}\right)$.

\subsection{Upper limits}

Searches for many other molecular species have been unsuccessfully conducted in various spectral ranges, notably in the UV and in the millimeter. In addition to that already mentioned on $\mathrm{CO}, \mathrm{H}_{2} \mathrm{~S}, \mathrm{PH}_{3}$, and $\mathrm{C}_{2} \mathrm{H}_{2}$, upper limits are available on $\mathrm{CH}_{3} \mathrm{OH}, \mathrm{H}_{2} \mathrm{CO}$, $\mathrm{SO}_{2}, \mathrm{SiO}, \mathrm{SO}, \mathrm{HC}_{3} \mathrm{~N}, \mathrm{CH}_{3} \mathrm{CN}, \mathrm{C}_{6} \mathrm{H}_{6}$, and $\mathrm{CO}^{+}$(see Noll et. al. 1995, Lellouch et al. 1995, 

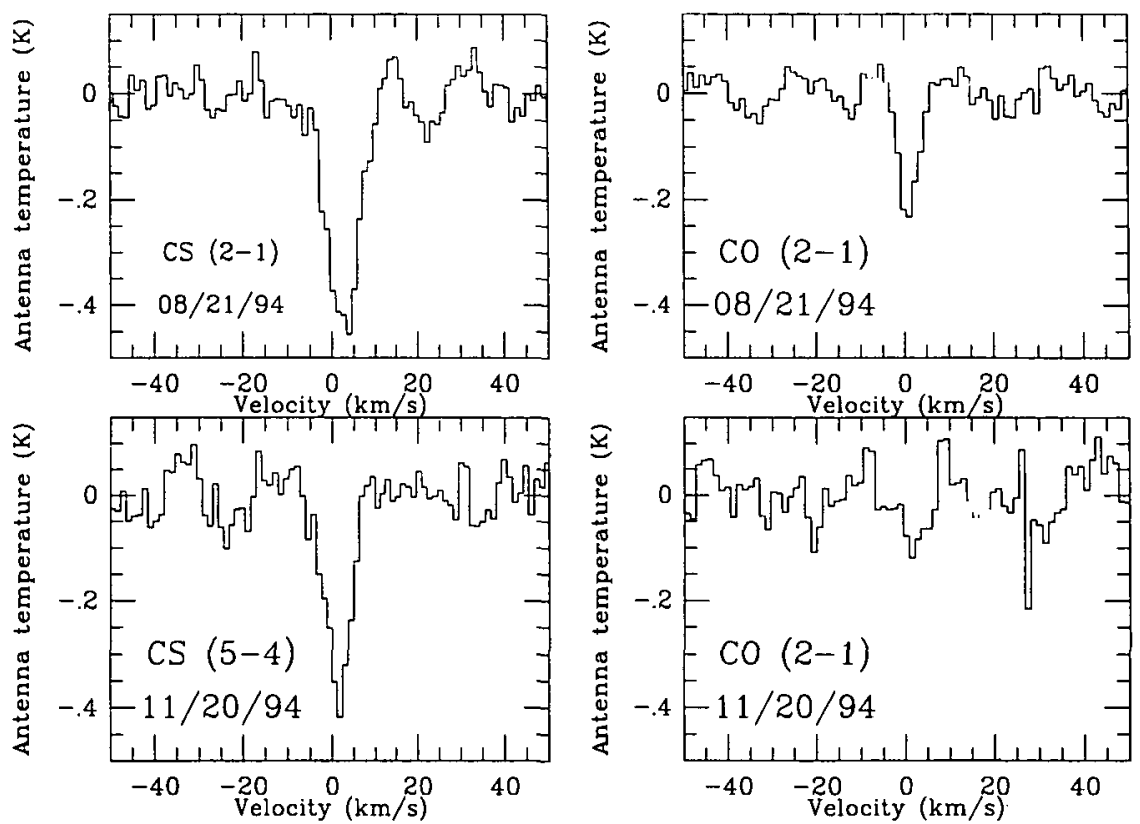

Figure 13. Long-term evolution of the CS (5-4) and CO (2-1) rotational lines, as observed from IRAM-30 m. Note the strong decrease of the CO (2-1) line. Courtesy R. Moreno.

Atreya et al. 1995, Yelle and McGrath 1996). Rather than give the associated values here, it seems more useful to mention that the derivation of these upper limits is generally based on a priori assumptions on the vertical distribution of the species. For example, the upper limits derived from the millimeter wave observations of Lellouch et al. (1995) implicitly assumed that the corresponding species are colocated with $\mathrm{CO}$ at $\mathrm{p} \leq 0.3 \mathrm{mbar}$. This assumption is probably valid for species that are directly produced by shock chemistry, but certainly not for molecules that result from subsequent photochemistry or that at updrafted by vertical mixing associated with the impacts. The case of $\mathrm{H}_{2} \mathrm{~S}$ is instructive, as the "raw" upper limit placed by Lellouch et al. $\left(3 \times 10^{-6} \mathrm{~g} \mathrm{~cm}^{-2}\right)$ is several times smaller than the actual column integrated mass of $\mathrm{H}_{2} \mathrm{~S}$ claimed by Yelle and McGrath (1995) $\left(2.5 \times 10^{-5} \mathrm{~g} \mathrm{~cm}^{-2}\right)$. However, as mentioned in Sec. 3.3, the Yelle and McGrath distribution for $\mathrm{H}_{2} \mathrm{~S}$ is, in fact, perfectly consistent with its non-detection at millimeter wavelengths. Also, in the case of photochemical species, simple assumptions on their vertical distributions (e.g., uniform mixing) may not be very meaningful in the context of photochemical models of these species. Therefore, underlying assumptions must be carefully examined before drawing too firm conclusions from the upper limits. With these caveats in mind, the most significant negative result is perhaps the non-detection of $\mathrm{SO}_{2}$ at UV wavelengths. Under the plausible hypothesis that $\mathrm{SO}_{2}$ has the same altitude distribution as $\mathrm{CS}_{2}$, Yelle and McGrath (1996) infer an upper limit of $\sim 10^{15} \mathrm{~cm}^{-2}$, implying that less $\mathrm{SO}_{2}$ is produced than $\mathrm{CS}_{2}$.

\section{Long-term evolution}

Observations of Jupiter at millimeter, infrared (10 micron) and UV wavelengths, performed during the weeks and months following the SL9 impacts, have provided insight on the chemical evolution of Jupiter's stratosphere on a long timescale. Analysis of these 
data is often incomplete at the date this review is written (November 1995), but a number of qualitative conclusions can still be drawn.

\subsection{UV spectrum}

HST/FOS observations of Jupiter at the impact latitude and near the central meridian were performed on March 3, April 7, and again in September 1995 (McGrath et al. 1995). These spectra show a number of interesting features: (i) the continuum reflectivity is back to its pre-impact value, (ii) $\mathrm{NH}_{3}$ bands dominate the spectrum at $1800-2200 \hat{\AA}$, (iii) these bands do not show any significant variability over a $4^{\circ}$ latitude range, and (iv) $\mathrm{CS}_{2}$ bands are still present, although much weaker than during the impact week. Since tropospheric ammonia bands are normally visible in low emission angle spectra of Jupiter (see Sec. 4.1.1.), a detailed modelling of these observations will be needed to deduce whether some ammonia was still present in Jupiter's stratosphere 8 and 14 months after the impacts.

\subsection{0-micron spectrum}

IRTF/IRSHELL 10-micron observations of Jupiter's southern hemisphere were performed on May 15-18, 1995. These observations show: (i) no sign of $\mathrm{NH}_{3}$ emission, implying an ammonia column density $\leq 10^{16} \mathrm{~cm}^{-2}$ above 40 mbar (i.e., 30 times less than in July 1994) (Bézard et al. 1995b), and (ii) strong persistent HCN emission, with a latitudinal distribution extending roughly from $20^{\circ} \mathrm{S}$ to $60^{\circ} \mathrm{S}$ (comparable to that of aerosol particles at the same time), and a heterogeneous longitudinal distribution at a $1.5^{\prime \prime}$ spatial resolution, with maximum HCN column densities of $\sim 5 \times 10^{15} \mathrm{~cm}^{-2}$ (Griffith et al. 1995). The total HCN mass seen in these data is $\geq 10$ times larger than that created by a large, K-sized, impact, suggesting a possible photochemical production of HCN after the impacts week.

Finally, Fast et al. (1995b) report that ammonia stratospheric emission is at most marginally seen in $10 \mu \mathrm{m}$ heterodyne observations performed in April 1995, suggesting an ammonia stratospheric mixing ratio of $10^{-10}$ or less.

\subsection{Millimeter-wave spectrum}

The millimeter-wave lines have dramatically changed since the impact period. CO, CS and $\mathrm{HCN}$ were originally observed as strong emission lines of comparable intensity, typically $40 \mathrm{~K}$ in brightness temperature. (OCS was detected only on one occasion and was weaker; the non-detection of OCS on August 19 is consistent with an expected photochemical decay by a factor $\sim 3$ over 1 month; Moses et al. 1995c). The contrast of the $\mathrm{CO}(2-1)$ and $\mathrm{CS}(5-4)$ lines decreased over the week following the impacts until around July 28, when the lines turned into absorption (Lellouch et al. 1995). Monitoring of these absorption lines at IRAM-30m on a week/month basis showed that, on the large impact sites, the CS absorption increased to reach a steady depth of $\sim 0.6 \mathrm{~K}$ (in antenna temperature), while the $\mathrm{CO}$ absorption reached a maximum of $\sim 0.3 \mathrm{~K}$ mid-August 1994, then decreased to a current depth of $\sim 0.15 \mathrm{~K}$ (Moreno et al. 1995; Fig. 13). CS is now observed also in its (3-2) line at $147 \mathrm{GHz}$. JCMT observations performed since September 1994 show that the HCN (4-3) line has a similar behavior as CS (Matthews et al. 1995). The turnover to the absorption regime must primarily be a temperature effect, with Jupiter's thermal profile in the 0.1-0.01 mbar region probably becoming colder than in pre-impact conditions (Lellouch et al. 1995, Conrath, this volume). The increase of absorption line contrast is certainly due to an increase of the emitting area in the telescope beam (the observations themselves show evidence for horizontal spreading of the material). More relevant to this review is the question of the peculiar evolution of the 
$\mathrm{CO}$ line. The comparison of $\mathrm{CO}$ and HCN is particularly puzzling, as these two species were probably formed at the same level and their vertical distribution must have followed the same evolution. As the observations have not been modelled yet, only qualitative suggestions can be proposed for the time being. Three explanations may be considered:

- Instrumental effect. If the CO line was less optically thick than the other two just after impact, it may now have become optically thin while the CS and HCN lines may still be optically thick. In the model presented in Sec. 2.2.2, the CO line has an optical depth of $\sim 9$. A complete modelling of the CS and HCN emission lines is necessary to test this possibility.

- Chemical effect with the increase of HCN and CS. If the CO column density is assumed to remain constant with time, the decrease of the $\mathrm{CO}$ line must be due to a combination of temperature and $\mathrm{CO}$ vertical profile evolution. As the same must happen for HCN and CS, the fact that the HCN and CS lines are still very strong implies that fresh amounts of $\mathrm{HCN}$ and CS must have been created well after the impact period, perhaps from $\mathrm{NH}_{3}$ and $\mathrm{N}_{2}$ (via cosmic ray impact) and from $\mathrm{CS}_{2}$ and $\mathrm{S}_{2}$, respectively. This hypothesis may seem viable as the initial mass of $\mathrm{NH}_{3}$ (and $\mathrm{N}_{2}$ ?) is larger than that of HCN (see Table 1); similarly, enough sulfur might be originally available in $\mathrm{S}_{2}$ to appreciably modify the CS amount with time. For HCN, recent photochemical models (Moses et al. 1995c) suggest that the HCN mass may increase by a factor about 2 over the initial abundance after 1 year.

- Chemical effect with a decrease of CO. If the HCN and CS are assumed approximately constant with time, the decrease of the $\mathrm{CO}$ line could be due to a true decrease of the $\mathrm{CO}$ mass. However, $\mathrm{CO}$ is notoriously stable and a mechanism must be found. $\mathrm{OH}$ radicals, liberated from the photolysis of $\mathrm{H}_{2} \mathrm{O}$ on a month timescale may react with and consume $\mathrm{CO}$ molecules (as is the case in Mars and Venus), but again, photochemical models suggest this effect is negligible (J. Moses, priv. comm.)

Recent analyses of the millimeter lines (Moreno et al. 1995, Matthews et al. 1995) suggest the following column densities 6-12 months after impact: CS: $5 \times 10^{14} \mathrm{~cm}^{-2}$; HCN: $1 \times 10^{15} \mathrm{~cm}^{-2}$; CO: $2.5 \times 10^{16} \mathrm{~cm}^{-2}$, i.e., 10,10 , and 160 times less than measured in July 1994 for the large impacts. The total extent of the chemical perturbation (from $10^{\circ} \mathrm{S}$ to $80^{\circ} \mathrm{S}$ ) is $\sim 100$ times larger than the total area of the fresh sites. Thus the total CS and HCN masses have remained approximately constant (or have moderately increased) since July 1994, while the total CO mass may have decreased by a factor 10. This would tentatively favor the third explanation.

\section{Synthesis and conclusions}

The observation of many molecular species in the plume/splashback phases and at the impact sites, most of which had never been observed in Jupiter before, provide a fairly detailed picture of the chemistry induced by the SL9 impacts. From the synthesis of the observations, one can draw several conclusions:

(a) All new or enhanced species were detected in the stratosphere or the thermosphere of Jupiter. There is no evidence for any variation of the chemical composition of the troposphere.

(b) Although the vertical distribution of some molecules is still uncertain $\left(\mathrm{NH}_{3}, \mathrm{C}_{2} \mathrm{H}_{4}\right)$, the observations suggest a distinction between two types of species (Yelle and McGrath 1996):

- Those present at pressures less than (0.1-1) mbar. These include $\mathrm{CO}, \mathrm{H}_{2} \mathrm{O}, \mathrm{S}_{2}$, $\mathrm{HCN}, \mathrm{CS}, \mathrm{OCS}, \mathrm{CS}_{2}, \mathrm{C}_{2} \mathrm{H}_{4}$, and a fraction of $\mathrm{NH}_{3}$ ). Since fallback models (Zahnle 


$\begin{array}{cc}\text { Species } & \text { Total mass }(\mathrm{g}) \\ \mathrm{CO} & 2.5 \times 10^{14} \\ \mathrm{H}_{2} \mathrm{O} & \geq 2 \times 10^{12} \\ \mathrm{~S}_{2} & 7 \times 10^{11} \\ \mathrm{CS}_{2} & 1.5 \times 10^{11} \\ \mathrm{CS} & 5 \times 10^{11} \\ \mathrm{OCS} & 3 \times 10^{12} \\ \mathrm{HCN} & 6 \times 10^{11} \\ \mathrm{C}_{2} \mathrm{H}_{4} & 3 \times 10^{12} \\ \mathrm{NH}_{3} & 1 \times 10^{13} \\ \mathrm{PH}_{3} & ? ? \\ \mathrm{H}_{2} \mathrm{~S} & ? ?\end{array}$

TABLE 1. "Baseline" masses for detected molecular species

et al. 1995, Zahnle, this volume) predict that plume material re-enters the atmosphere at pressure levels of $10-100 \mu \mathrm{bar}$, the simplest interpretation is that these species are either formed locally during plume re-entry or formed during the primary explosion, transported by the plume, and deposited in the upper stratosphere. Shock chemistry is in fact expected to take place both at explosion and during plume splashback. One aspect which remains to be explained is the apparent segregation of $\mathrm{S}_{2}$ (present at $\mathrm{p} \leq 0.1 \mu \mathrm{bar}$ only) with respect to the other species.

- Those present at $\mathrm{p} \geq(1-10)$ mbar, must result from upwelling caused by the heating of jovian air generated by the impacts. Ammonia would be the prime example. This could be also the case for $\mathrm{PH}_{3}$ and $\mathrm{H}_{2} \mathrm{~S}$, if their detections are confirmed.

Note that ammonia apparently falls into the two categories. Carbon monosulfide is also an exception as a significant fraction of it may result from photochemistry rather than from hot/shock chemistry.

(c) Abundance determinations are available for most observations, especially for the "site" measurements. Except in the case of a number of plume/splashback observations, the comparison of retrieved abundances indicates an overall reasonable consistency. Plausible explanations can be found to account for the remaining discrepancies (e.g., CS, $\mathrm{NH}_{3}$ ) and future intercomparisons will allow to refine estimations. In almost no case (except for water) have the variations of molecular abundances from fragment to fragment been investigated. Future studies may allow to correlate the mass of the chemicals produced by the individual impact with the brightness/mass of the fragments. For the time being, "baseline" masses for the different molecular species, valid for a "large" impactor (i.e., G, K), are gathered in Table 1. Some points must be made regarding Table 1: (i) the value for $\mathrm{H}_{2} \mathrm{O}$ can be taken as a reasonably close lower limit (Sec. 1.1), (ii) the $\mathrm{S}_{2}$ and $\mathrm{CS}_{2}$ masses were obtained by using the column densities derived from HST/FOS and assuming an areal extent identical to that of $\mathrm{CO}$. Whether $\mathrm{S}_{2}$ is indeed more abundant than CS is an important question to test the possibility that CS is photochemically produced from $\mathrm{S}_{2}$, (iii) the question mark for $\mathrm{H}_{2} \mathrm{~S}$ and $\mathrm{PH}_{3}$ indicates that the detection is tentative. No mass estimate can be given since the depth to which they extend is unknown. For reasons discussed in Sec. 5.3, upper limits are not included in this Table.

(d) The molecular masses indicated in Table 1 imply $\sim 1.5 \times 10^{14} \mathrm{~g}$ of atomic $\mathrm{O}$ and $\sim 2.7 \times 10^{12} \mathrm{~g}$ of atomic $\mathrm{S}$ (excluding $\mathrm{H}_{2} \mathrm{~S}$ ). The mass of atomic $\mathrm{N}$ is $\sim 3 \times 10^{11} \mathrm{~g}$ if only $\mathrm{HCN}$ is included and $\sim 1 \times 10^{13} \mathrm{~g}$ if $\mathrm{NH}_{3}$ is considered. Assuming relative atomic abundances for the cometary fragments similar to those measured in comet $\mathrm{P} / \mathrm{Halley}$ 
(Jessberger et al. 1986), a $1.5 \times 10^{14} \mathrm{~g}$ mass of $\mathrm{O}$ is available in a $\sim 3 \times 10^{14} \mathrm{~g}$ fragment. Tidal disruption models show that the parent comet was about $1.5 \mathrm{~km}$ diameter and had a density of $\sim 0.6 \pm 0.1 \mathrm{~g} \mathrm{~cm}^{-3}$ (Asphang and Benz 1995, Solem 1994), i.e., a mass of $\sim 10^{15} \mathrm{~g}$. The largest fragments may be assigned $1 / 8$ of the total mass, or $1.3 \times 10^{14} \mathrm{~g}$. This is 2.5 times less than required from the observed oxygen mass, but the agreement may be considered as reasonable given the various uncertainties. In addition, the $\mathrm{O} / \mathrm{S}$ ratio implied by the measurements is nominally 56 , in plausible consistency with the value for comet $\mathrm{P} / \mathrm{Halley}$ (14) (especially given the uncertainty in the mass of $\mathrm{S}_{2}$ ). For $\mathrm{O} / \mathrm{N}$, the observations give a value of 500 if only $\mathrm{HCN}$ is considered, and 15 if ammonia is included, while the P/Halley value is 25 . Therefore, the amounts of $\mathrm{O}, \mathrm{N}$, and $\mathrm{S}$ present in a large fragment can probably account for all the observed molecular abundances, so on this basis only, one might conclude that the shock chemistry involves jovian $\mathrm{H}$ and $\mathrm{C}$ (from $\mathrm{H}_{2}$ and $\mathrm{CH}_{4}$ ) and purely cometary $\mathrm{O}, \mathrm{N}$, and $\mathrm{S}$. This view is probably incorrect, however, notably because most entry models (see Zahnle, Mac Low, Crawford, this volume) predict that km-size fragments penetrate down to or below the $\mathrm{NH}_{4} \mathrm{SH}$ cloud at 2 bars, so that jovian sulfur must be involved in the chemistry. Another remark that can be made is that if, as suggested by some observations, most of the ammonia is updrafted from Jupiter's troposphere, then $\mathrm{NH}_{3}$ must not be included when calculating the observed $\mathrm{O} / \mathrm{N}$ ratio. In this case, the comparison with the $\mathrm{P} /$ Halley value indicates that another important $\mathrm{N}$-bearing species has been produced but not observed, presumably $\mathrm{N}_{2}$. Finally, if one accepts Yelle and McGrath's (1996) conclusion that $\mathrm{H}_{2} \mathrm{~S}$ is detected in the HST/FOS spectra, then their observed N/S ratio is approximately 2, in reasonable agreement with but somewhat smaller than the solar value (about 6; Grevesse and Anders 1991), which might suggest that sulfur is more enriched than nitrogen below the jovian $\mathrm{NH}_{4} \mathrm{SH}$ clouds.

(e) The observed presence of water and the absence of $\mathrm{SO}_{2}$ is a problem for models (see Zahnle, this volume), because shock chemistry in an oxygen-rich environment $(\mathrm{O} / \mathrm{C} \geq 1)$ leads to abundant production of $\mathrm{H}_{2} \mathrm{O}$ and $\mathrm{SO}_{2}$, while neither $\mathrm{H}_{2} \mathrm{O}$ nor $\mathrm{SO}_{2}$ is produced in oxygen-poor conditions $(\mathrm{O} / \mathrm{C} \leq 1)$. A possibility is that the observed $\mathrm{H}_{2} \mathrm{O}$ results from cometary water that survived the explosion. The case for jovian $\mathrm{H}_{2} \mathrm{O}$ excavated from below the water cloud does not seem very likely as this region has $\mathrm{O} / \mathrm{C} \sim 2-10$, while the production of $C S, C S_{2}$ and $\mathrm{HCN}$ requires $\mathrm{O} / \mathrm{C} \leq 1$ in the shocked jovian air (Zahnle, this volume).

$(f)$ The ammonia stratospheric mixing ratio is of order $0.5-1 \times 10^{-7}$ (at a $\sim 5000 \mathrm{~km}$ spatial resolution), which is the nominal (pre-impact) value at $\sim 0.2$ bar, and well below the abundance below the clouds $\left(\sim 3 \times 10^{-4}\right)$. If one admits that the large fragments exploded below the clouds, this suggests some very large horizontal dilution of the convected tropospheric jovian air at stratospheric levels.

(g) There is observational evidence for photochemistry occuring after the impacts. This is attested in particular by the decay of $\mathrm{S}_{2}, \mathrm{CS}_{2}, \mathrm{NH}_{3}$ and the possible increase of CS on a week or month timescale.

Illuminating discussions with many colleagues are acknowledged, particularly S. Atreya, A. L. Betz, B. Bézard, J. Caldwell, B. Conrath, D. Crisp, C. Griffith, D. Hunten, T. Kostiuk, V. Meadows, J. Moses, R. Moreno, K. Noll, A. Sprague, R. Yelle, and K. Zahnle. This paper is dedicated to the memory of Jan Rosenqvist, deceased on July 20, 1995. 


\section{REFERENCES}

ANICICH, V. G., \& Huntress, W. T. 1986 A survey of bimolecular ion-molecule reactions for use in modeling the chemistry of planetary atmospheres, cometary comae, and interstellar clouds. Astrophys. J. Suppl. Ser. 62, 553-672.

AsphaUg, E., \& BENZ, W. 1995 The tidal disruption of strengthless bodies: lessons from comet Shoemaker-Levy 9. Icarus (submitted).

AtreyA, S. K. 1986 Atmospheres and ionospheres of the outer planets and their satellites. In Physics and Chemistry in Space, Vol. 15, (eds. L. J. Lanzerotti and D. Stöffler). SpringerVerlag.

Atreya, S. K., Edgington, S., Trafton, L. M., Caldwell, J. J., Noll, K. S. \& Weaver, H. A. 1995 Abundances of ammonia and carbon disulfide in the Jovian stratosphere following the impact of comet Shoemaker-Levy 9. Geophys. Res. Lett. 22, 1625-1628.

Betz, A. L., Boreiko, R. T., Bester, M., Danchi, W. C., \& Hale D. D. 1995 Stratospheric ammonia in Jupiter after the impact of comet Shoemaker-Levy 9., Bull. Amer. Astron. Soc., 26, 1590-1591.

BÉZARD, B., GrifFith, C. A., LACY, J., \& OWEN, T. 1995a Non-detection of hydrogen cyanide on Jupiter. Icarus, 118, 384-391.

Bézard, B., Griffith, C. A., Greathouse, T., Kelly, D., Lacy, J., \& Orton, G. 1995b Jupiter ten months after the collision of comet SL9: stratospheric temperatures and ammonia distribution Bull. Amer. Astron. Soc. 27, 72.

Bézard, B., Griffith, C. A., Greathouse, T., Kelly, D., Lacy, J., \& Orton, G. 1996 Infrared spectral images of comet SL9 impact sites: temperature and HCN retrievals. Icarus, submitted.

Bjoraker, G. L., Strolovy, S. R., Herter, T. L., Gull, G. \& Pirger, B. E. 1995a Detection of water after the collision of fragments $G$ and $K$ of comet Shoemaker-Levy 9 with Jupiter 1995. Science, submitted.

Bjoraker, G. L., Herter, T., Gull, G., Stolovy, S. \& Pirger, B. 1995b Detection of water in the "splash" of fragments G and K of comet Shoemaker-Levy 9. In Abstracts for IAU colloquium 156: The collision of comet P/Shoemaker-Levy 9 and Jupiter, p. 8.

Bockelée-Morvan, D., Lellouch, E., Colom, O., Paubert, G., Moreno, R., Festou, M., Despois, D., Sievers, A., Crovisier, J., Gautier, D. \& Marten, A. 1995 Millimetre observations of the Shoemaker-Levy 9 impacts on Jupiter in July 1994 at the IRAM and SEST telescopes: CO, CS and OCS. In Proceedings of European SL-9/Jupiter Workshop (eds. R. West and H. Bohnhardt), pp. 251-255.

Brooke, T. Y., Orton, G. S., Crisp, D., Friedson, A. J. \& Bjoraker, G. 1995 Nearinfrared spectroscopy of the Shoemaker-Levy 9 impact sites with UKIRT: CO emission from the L site. In Abstracts for IAU colloquium 156: The collision of comet P/Shoemaker-Levy 9 and Jupiter, p. 12.

Caldwell, J. \& 9 CO-Authors. 1995 Upper limits on $\mathrm{SiO}, \mathrm{H}_{2} \mathrm{~S}, \mathrm{C}_{2} \mathrm{H}_{2}$ and $\mathrm{H}_{2} \mathrm{O}$ on Jupiter from SL-9. Bull. Amer. Astron. Soc. 27, 64.

Carlson, R. W., Weissman, P. R., Hui, J., Segura, M., Smythe, W. D., Baines, K. H., Johnson, T. V., Drossart, P., Encrenaz, T., Leader, F. \& Mehlman, R. 1995a Some timing and spectral aspects of the $\mathrm{G}$ and $\mathrm{R}$ collision events as observed by the Galileo near-infrared mapping spectrometer. In Proceedings of European SL-9/Jupiter Workshop (ed. R. West and H. Bohnhardt), pp. 69-74.

Carlson, R. W., Weissman, P. R., Hui, J., Segura, M., Smythe, W. D., Baines, K. H., Johnson, T. V., Drossart, P., Encrenaz, T., Leader, F. \& Mehlman, R. 1995b Galileo infrared observations of the Shoemaker-Levy $9 \mathrm{G}$ and $\mathrm{R}$ fireballs and flash. In Abstracts for IAU colloquium 156: The collision of comet. P/Shoemaker-Levy 9 and Jupiter, p. 15.

Carlson, R. W., Weissman, P. R., Hui, J., Segura, M., T. V., Drossart, P. \& EnCRENAZ, T. 1995c Galileo infrared observations of the Shoemaker-Levy $9 \mathrm{G}$ and $\mathrm{R}$ splash phases. Bull. Amer. Astron. Soc. 27, 66. 
Carpenter, K. G., McGrath, M. A., Yelle, R. V., Noll, K. S. \& Weaver H. A. 1995 Formation of atomic emission lines in the atmosphere of Jupiter after the comet ShoemakerLevy $9 \mathrm{~S}$ impact. Bull. Amer. Astron. Soc. 27, 64.

Conrath, B. J., Gierasch, P. J., Hayward, T., McGhee, C. Nicholson, P. D. \& Van ClEVE, J. 1995 Palomar mid-infrared spectroscopic observations of comet ShoemakerLevy 9 impact sites. In Abstracts for $1 A U$ colloquium 156: The collision of comet P/Shoemaker-Levy 9 and Jupiter, p. 24.

Cosmovici, C. B., Montebugnoli S., Pogrebenko, S. \& Colom, P. 1995 Water MASER detection at $22 \mathrm{GHz}$ after the SL-9/Jupiter collision. Bull. Amer. Astron. Soc. 27, 79.

CRISP, D. \& MEADows, V. 1995 Near-infrared imaging spectroscopy of the impacts of SL9 fragments C, D, G, K, N, R, V, and W with Jupiter. In Abstracts for IAU colloquium 156: The collision of comet P/Shoemaker-Levy 9 and Jupiter, p. 25.

Drossart, P., Bézard, B., Atreya, S. K., Bishop, J., Waite, Jr., J. H. \& Boice, D. 1993 Thermal profiles in the auroral regions of Jupiter. J. Geophys. Res. 98, 18803-18811.

Fast, K. E., Kostiuk, T., Espenak, F., Zipoy, D., Buhl, D., Livengood, T. A., BjoR.AKER, G., RomANI, P. \& GoldsteIN, J. J. 1995a Infrared heterodyne observations of $\mathrm{NH}_{3}$ and $\mathrm{C}_{2} \mathrm{H}_{6}$ after the collision of comet $\mathrm{P} /$ Shoemaker-Levy 9 with Jupiter. In IAU colloquium 156: The collision of comet P/Shoemaker-Levy 9 and Jupiter, p. 25.

Fast, K. E., Livengood, T. A., Kostiuk, T., Buhl, D., Espenak, F., B.oraker, G. L., Romani, P. N., Jennings, D. E., Sada, P., Zipoy, D., Goldstein, J. J. \& Hewegama, T. 1995b $\mathrm{NH}_{3}$ in Jupiter's stratosphere within the year following the SL9 impacts Bull. Amer. Astron. Soc. 27, 72.

Feldman, P. D., Durrance, S. T. \& Davidsen, A. F. 1995 Far-ultraviolet spectroscopy of Venus and Mars at $4 \AA$ resolution with the Hopkins Ultraviolet Telescope on Astro-2 Bull. Amer. Astron. Soc. 27, 1079.

Fitzsimmons, A., Little, J. E., Wlaton, N., Catchpole, Andrews, P. J., Williams, I. P., Harlaftis, E. \& RUdD, P. 1995 Optical imaging and spectroscopy of the impact plumes on Jupiter. In Proceedings of European SL-9/Jupiter Workshop (ed. R. West and H. Bohnhardt) pp. 197-201.

Gladstone, G. R., Allen, M. \& YUng, Y. L. 1996 Hydrocarbon photochemistry in the upper atmosphere of Jupiter. Icarus, 119, 1-52.

Grevesse, N, \& ANDERs, E. 1991 Solar element abundances. In Solar interior and atmosphere (eds. A. N. Cox, W. C. Livingston and M. S. Matthews), pp. 1227-1234. The University of Arizona Press.

Griffith, C. A., Bézard, B., Greathouse, T., Kelly, D., Lacy, J., \& Orton, G. 1995 Jupiter ten months after the collision of comet SL9: spectral maps of $\mathrm{HCN}$ and $\mathrm{NH}_{3}$ Bull. Amer. Astron. Soc. 27, 72.

Griffith, C. A., Bézard, B., Greathouse, T., Kelly, D., Lacy, J., \& Noll, K. 1996 Infrared spectral images of comet SL9 impact sites: spatial and vertical distributions of ammonia, ethylene, and the enhanced $10 \mu \mathrm{m}$ continuum emission, 21 hours, 6 and 12 days following collision. Icarus, submitted.

HAMMEL, H. B. \& 16 CO-AUTHORS. 1995 HST imaging of atmospheric phenomena created by the impact of comet Shoemaker-Levy 9. Science 267, 1288-1296.

HARRIS, W., M. \& 15 CO-AUTHORS. 1995 A comprehensive IUE study of UV phenomena related to the collision of comet Shoemaker-Levy 9 (1993e) with Jupiter. Science, submitted.

Herbst, T. M., Hamilton, D. P., Boehnhardt, H. \& Ortiz-Moreno, J.-L. 1995a SL-9 impact imaging, spectroscopy and long-term monitoring from the Calar Alto Observatory. In Proceedings of European SL-9/Jupiter Workshop (eds. R. West and H. Bohnhardt) pp. $119-122$.

Herbst, T. M., Hamilton, D. P., Boehnhardt, H. \& Ortiz-Moreno, J.-L. 1995b Nearinfrared spectroscopy and long-term monitoring of the SL-9 impacts. In IAU colloquium 156: The collision of comet. $P /$ Shoemaker-Levy 9 and Jupiter, p. 50.

Hooker, W. J. \& MillikaN, R. C. 1963 Shock-tube study of vibration relaxation in carbon monoxide for the fundamental and the first overtone. J. Chem. Phys. 38, 214-220. 
Jessberger, E. K. \& KIssel, J. 1986 Chemical properties of cometary dust and a note on carbon isotopes. In Comets in the Post Halley Era, Vol. 2, (eds. R. L. Newburn, Jr., M. Neugebauer and J. Rahe), pp. 1075-1092. Kluwer Academic.

Kim, S.-J., Caldwell, J., Rivolo, A. R., Wagener, R. \& Orton, G. S. 1985 Infrared polar brightening on Jupiter, III, Spectrometry from the Voyager 1 IRIS experiment. Icarus 64, $233-248$.

Kim, S. J., Ruiz, M., RiEke, G. H. \& Rieke, J. 1995 Thermal history of the R impact flare of comet Shoemaker-Levy 9. Bull. Amer. Astron. Soc. 27, 66.

Knacke, R. F., Fajardo-Acosta, S. B., Geballe, T. R., \& Noll, K. S. 1995 Near-infrared spectroscopy of the R site of comet Shoemaker-Levy 9. In IAU colloquium 156: The collision of comet P/Shoemaker-Levy 9 and Jupiter, p. 59.

Kostiuk, T., Romani, P., Espenak, F., Livengood, T. \& Goldstein, J. J. 1993 Temperatures and abundances in the Jovian auroral stratosphere, 2, Ethylene as a probe of the microbar region. J. Geophys. Res. 98, 18823-18830.

Kostiuk, T., Espenak, F., Buhl, D., Romani, P. N., Fast, K. E., Livengood, T. A., Zipoy, D., Hewegama, T. \& Goldstein, J. J. 1995 Altitude distribution of ammonia deposited in Jupiter's stratosphere by the SL9 impacts. Bull. Amer. Astron. Soc. 27, 72.

LELLOUCH, E. \& 13 CO-AUTHORS 1995 Chemical and thermal response of Jupiter's atmosphere following the impact of comet Shoemaker-Levy 9. Nature 373, 592-595.

Livengood, T. A., Kostiuk, T., Espenak, F. \& Goldstein, J. J. 1993 Temperatures and abundances in the Jovian auroral stratosphere, 1, Ethane as a probe of the millibar region. J. Geophys. Res. 98, 18813-18822.

Maillard, J.-P., Drossart, P., Bézard, B., De Bergh, C., Lellouch, E., Marten, A., Caldwell, J., Hilico, J.-C. \& Atreya, S. K. 1995 Methane and carbon monoxide einfrared emissions observed at the Canada-France-Hawaii telescope during the collision of comet SL-9 with Jupiter. Geophys. Res. Lett. 22, 1573-1576.

MARTEN, A. \& 16 CO-AUTHORS. 1995 The collision of the comet Shoemaker-Levy 9 with Jupiter: detection and evolution of $\mathrm{HCN}$ in the stratosphere of the planet. Geophys. Res. Lett. 22, 1589-1592.

Matthews, H. E., Marten, A., Griffin, M. J., Owen, T., \& Gautier, D. 1995 JCMT observations of long-lived molecules on Jupiter in the aftermath of the comet ShoemakerLevy 9 collision Bull. Amer. Astron. Soc. 27, 67.

McGrath, M. A., Yelle, R. V., Noll, K. S., Weaver, H. A. \& Smith, T. E. 1995 Hubble Space Telescope spectroscopic observations of the jovian atmosphere following the SL9 impacts. Bull. Amer. Astron. Soc. 27, 64.

MEADows, V. \& CRISP, D. 1995a Impact plume composition from near-infrared spectroscopy. In Proceedings of European SL-9/Jupiter Workshop (eds. R. West and H. Bohnhardt) pp. 239-244.

MEadows, V. \& CRISP, D. 1995b Near-infrared imaging spectroscopy of the impacts of SL9 fragments C, D, G, K, N, R, V, and W with Jupiter. Bull. Amer. Astron. Soc. 27, 73.

Montebugnoli, S. \& 16 CO-AUTHors 1995 Detection of the 22-GHz line of water during and after the SL-9/Jupiter event. In Proceedings of European SL-9/Jupiter Workshop (eds. R. West and H. Bohnhardt) pp. 261-266.

Moreno, R., Marten, A., Lellouch E., Paubert, G. \& Wild, W. 1995 Long-term evolution of $\mathrm{CO}$ and $\mathrm{CS}$ in the Jupiter stratosphere after the comet Shoemaker-Levy 9 collision: millimeter observations with the IRAM 30-m telescope. Bull. Amer. Astron. Soc. 27, 75.

Moses, J. I., Allen, M. \& Gladstone, G. R. 1995a Post SL9 sulfur photochemistry on Jupiter. Geophys. Res, Lett. 22, 1597-1600.

Moses, J. I., Allen, M. \& Gladstone, G. R. 1995b Nitrogen and oxygen photochemistry following SL9. Geophys. Res. Lett. 22, 1601-1604.

Moses, J. I., Allen, M., Fegley, B., Jr. \& Gladstone, G. R. 1995c Photochemical evolution of the post-SL9 jovian stratosphere. Bull. Amer. Astron. Soc. 27, 65.

Noll, K. S., Knacke, R. F., Geballe, T. R. \& Tokunaga, A. T. 1995 The origin and vertical distribution of carbon monoxide on Jupiter. Astrophys. J. 324, 1210-1218. 
Noll, K. S., McGrath, M. A., Trafton, L. M., Atreya, S. K., Caldwell, J. J., Weaver, H. A., Yelle, R. V., BARnet, C. \& Edgington, S. 1995 HST spectroscopic observations of Jupiter after the collision of comet Shoemaker-Levy 9. Science 267, 1307-1313.

OrTon, G. \& 57 CO-AUTHOR.s. 1995 Collision of comet Shoemaker-Levy 9 with Jupiter observed by the NASA Infrared Telescope facility. Science 267, 1277-1282.

Roos-Serote, M., Barucci, A., Crovisier, J., Drossart, P., Fulchignoni, M., LeCACHEUX, J. \& Roques, F. 1995 Metallic emission lines during the impacts $\mathrm{L}$ and $\mathrm{Q}_{1}$ of comet P/Shoemaker-Levy 9 in Jupiter. Geophys. Res. Lett. 22, 1621-1624.

Ruiz, M., Rieke, G. H., Rieke, M. J., Means, D. \& Frawley, P. 1994 Near infrared spectroscopy of SL-9 impacts. Earth Moon Plan. 66, 91-98.

Solem, J. C. 1994. Density and size of comet Shoemaker-Levy 9 deduced from a tidal breakup model. Nature 370, 349-351.

Sprague, A. L., Buoraker, G. L., Hunten, D. M., Witteborn, F. C., Kozlowski, R. W. H. \& Wooden, D. H. 1996. Water brought into Jupiter's atmosphere by fragments $\mathrm{R}$ and $\mathrm{W}$ of comet SL-9. Icarus, in press.

Tokunaga, A. T., Beck, S. C., Geballe, T. R., Lacy, J. H., \& Serabyn, E. M. 1995 The detection of HCN on Jupiter. Icarus 48, 283-289.

Trafton, L. M., Atreya, S. K., McGrath, M. A., Gladstone, G. R., Caldwell, J. J., Noll, K. S., Weaver, H. A., Yelle, R. V., Barnet, C. \& Edgington, S. 1995 FUV spectra of SL9 impact site G with HST/GHRS. In IAU colloquium 156: The collision of comet $P /$ Shoemaker-Levy 9 and Jupiter, p. 112.

Wagener, R., Caldwell, J., Owen, T., Kim, S.-J., Encrenaz, T. \& Combes, M. 1985 The jovian stratosphere in the ultraviolet. Icarus 63, 222-236.

West, R. A., Karkoschka, E., Friedson, A. J., Seymour, M., Baines, K. H. \& Hammel, H. B. 1995 Impact debris particles in Jupiter's stratosphere. Science 267 1296-1301.

Yelle, R. V. \& MCGrath, M. A. 1995 Results from HST spectroscopy of the SL9 impact sites. Bull. Amer. Astron. Soc. 27, 64.

YelLe, R. V. \& MCGrath, M. A. 1996 Ultraviolet spectroscopy of the SL9 impact sites, I: The $175-230 \mathrm{~nm}$ region. Icarus, in press.

Zahnle, K., Mac Low, M.-M., Lodders, K., \& Fegley, Jr., B. F. 1995 Sulfur chemistry in the wave of comet Shoemaker-Levy 9. Geophys. Res. Lett. 22, 1593-1596. 